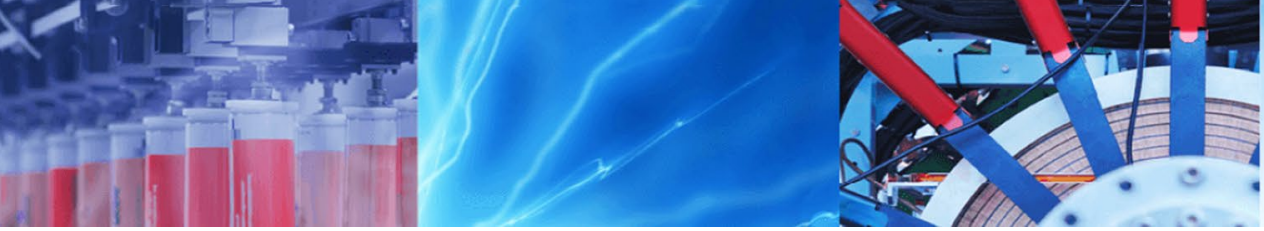

Review Paper

\title{
Clarifications of concepts concerning interplanar spacing in crystals with reference to recent publications
}

\author{
Ying Liu' ${ }^{1}$ Yue Liu' ${ }^{1} \cdot$ Michael G. B. Drew²
}

Received: 8 October 2019 / Accepted: 12 March 2020 / Published online: 25 March 2020

(c) Springer Nature Switzerland AG 2020

\begin{abstract}
Large number of papers are published each year on most areas of science, the majority being dependent on previously derived theories. It sometimes seems obvious that the authors have accepted the conventionally accepted theories that are the basis for their work without fully understanding all the details. We have reviewed several recent papers in the field of crystallography and found that several fundamental concepts have been applied in a manner that needs to be clarified. There are important principles imbedded in the conventional definition of Miller indices. Failure in understanding the advantages inherent within this definition has led in some recent publications to an improper redefinition of Miller indices and confusion between the concepts of lattice node and Wyckoff site and between symmetry equivalence and environment equivalence of points in lattice. Related problems involve the misleading claims that the node density among parallel lattice planes with the same Miller indices can be different, and also that lattice interplanar spacing can be different between adjacent parallel lattice planes. In this work, these problems have been clarified. A new method to calculate the relative lattice node density between planes with different Miller indices in a lattice has been developed. It illustrates that in a lattice, the node density of all the other planes with different Miller indices has already been determined if the node density of a particular plane with specified Miller indices is known. Related experimental results have also been discussed. The work shows that it is sometimes problematic for researchers to interpret experimental data via well-known theories without a full understanding of the basic science involved.
\end{abstract}

Keywords Miller indices $\cdot$ Wyckoff site $\cdot$ Lattice node $\cdot$ Lattice node density

\section{Introduction}

Reviews can be broadly divided into two types which are equally valuable to scientists. The first type provides a detailed organized survey from large amounts of experimental data available rather than offering theoretical insights. The second type concentrates on clarifying old theories or developing new ones using critiques of published materials. The latter category can be considered to be borderline between a research paper and a conventional review and could be called a research review. This work belongs to the latter type and focuses in detail on five recent articles [1-5] published in the field of crystallography.

There are very many papers published every year of which quite a number contain mistakes that originated from ignoring the rudiments of physics. Thus, research reviews are needed since any problematic work published in the scientific community deserves to be reconsidered in the light of fundamental theories, with the aim of clarifying obscurities, correcting inaccuracies, or gaining new understanding [6]. We have also focused on this kind of research and can offer many examples where as a consequence, new results and insights were obtained. For example, by challenging

\footnotetext{
$\triangle$ Yue Liu, yueliusd@163.com; Ying Liu, yingliusd@163.com; Michael G. B. Drew, m.g.b.drew@reading.ac.uk| ${ }^{1}$ College of Chemistry and Chemical engineering, Shenyang Normal University, Shenyang 110034, People's Republic of China. ${ }^{2}$ School of Chemistry, The University of Reading, Whiteknights, Reading RG6 6AD, UK.
} 
the correctness of Le Châtelier's principle, still dominant in the scientific community, theoretical reviews on the subject have provided novel rigorous mathematical methods to deal with complicated chemical equilibrium [7-11]. A systematic system for analyzing the chirality of octahedral chelate complexes has been established by reviewing the relevant published methods [12]. Two sets of equations for angles between direct and reciprocal vectors in crystallography and for symmetry operations in quantum theory by Euler's theorem are usually treated as unrelated equations, but we [13] have proved from a careful mathematical review that they are essentially the same though they appear in different scientific fields and different symbols are used for the same physical quantity. There are already many methods available to obtain the Russell-Saunders terms in atomic spectroscopy, and the topic might have been considered successfully concluded. But we have developed new methods which provided new insights $[14,15]$.

Most researchers are keen in reporting new experimental results and this can be achieved quickly and relatively easily with the resource of state-of-the-art equipment. A minority are more interested in using their experimental data to clarify current theories or develop new ones. This latter type of work can be difficult, time-consuming, and requires solid knowledge in science. However, the former type of research has become dominant nowadays with the result that reporting experimental results has become the norm and advances in theory are seldom to be found in the literature. The most obvious reason for this disparity is that experimental results with eye-catching presentations are often exciting and attractive; [16] and can therefore be considered as more important than theoretical work which can seem dull and too basic since real understanding usually originates from the rudiments of science established long before. For example, the passive film proposal for metal protection based on the anodic polarization has already entered textbooks because it seemed that a problem had been resolved and the solution was satisfactory. But the proposal has flaws which can be readily fixed by using the concepts of college physics [17]. A further example is the widely applied concept of reflection loss $R L$ in the field of material research but the currently accepted practices contradicts basic principles in physics [18-22]. As a result, theoretical gaps have developed between the published work of researchers in microwave absorbing materials and microwave engineers.

The imbalance between the aims of research work has developed presumably because most researchers believe publishing many papers on experimental results provides a clearer career path compared to theoretical work which can be incomplete and needs ratification from experiments [23, 24]. Thus, there is less enthusiasm for reviewing published experimental data [25] since to comprehend theoretical background needs a lot of previous training and it is hard work even to learn from textbooks. However, theoretical research can be worthwhile and generally helpful to the experimentalists. For example, based on the rudiments of crystal structure of ferrites, $[26,27]$ their synthetism has been optimized to achieve better results $[28,29]$. After theoretical considerations, the relationship between Fourier series expansion in mathematics and Hückel theory in chemistry has been revealed [30]. Lacking interest in fundamental theory can lead to problems in academic honesty [31-35] and mistakes [1-5, 7-11, 17-22] in publications, originating from the fact that researchers are, perhaps necessarily, too eager for the next publication.

In this current work, we consider aspects of the traditional field of crystallography. Within the well-developed traditional field, inventions are difficult to achieve nowadays but still possible. This work is concerned with recent papers [1-5] on the traditional concepts related to the definition of Miller indices, diffraction indices, Wyckoff site, asymmetric unit, node density, and interplanar spacing, etc. in crystal lattices.

Unit vectors in a reciprocal space are associated with stacks of planes in a crystal lattice. Two equivalent expressions for the magnitude of a unit vector in a reciprocal space, where the vector is related to interplanar spacing of parallel lattice planes, are presented in Sect. 2.1. The conventional Miller indices are closely related to interplanar spacing in lattices. Failure to realize the imbedded principles in the conventional definition of the indices has led to several misleading claims: (i) that the conventional definition of Miller indices is wrong and the indices (111) of an 1 -centered cell should be (222) [3]; (ii) that a new but misleading redefinition has been proposed in the claims [1] which already has some negative influence in the literature [36]; and (iii) that it is impossible to have different environments around positions connected by symmetry operations [3]. These problems are clarified in Sect. 2 with a detailed discussion involving the meaning and consequences of the definition of Miller indices. A clear difference between a node and a position of the atom in a crystal lattice is given, leading to show that two equivalent atomic sites may have different environments. It is emphasized that a set of Miller indices (unit vector in the reciprocal space) specifies a stack of lattice planes and not only one specified single plane. Another relevant point is the fact that lattice nodes should not be confused with Wyckoff sites. Wyckoff sites refer to the site symmetry of special points which has been used to specify the crystal structure without ambiguity. On the other hand, all lattice nodes by definition have the same environment related to translation, a fact which has been used to characterize a lattice and differentiate it from a crystal. The importance of this difference has been illustrated by examples. The relation between alternative unit cells in a lattice is also discussed. 
The redefinition [1] of Miller indices also leads to the misleading claim that in a lattice, the node density of a lattice plane can be different for an individual plane in a set of parallel lattice planes with the same Miller indices [4] and the related claim that interplanar spacing can be different for adjacent planes in a family of parallel lattice planes [5]. These two issues will be discussed in Sects. 3 and 4, respectively. The consequences of the redefinition of Miller indices have been illustrated by a variety of examples related to node density and interplanar spacing in lattice planes. In Sect. 3.4, a new method is provided to calculate the relative lattice node density between planes with different Miller indices in a three-dimensional lattice using fundamental principles. It is demonstrated that the node densities of lattice planes with different Miller indices can be determined if the node density of a particular lattice plane with specified Miller indices is known. The method conforms to the fact that the same lattice can be represented equally by many of the different non-conventional units. This general approach is contrasted with Fan's method [4]. It is shown that the approaches claimed as novel by Fan $[4,5]$ are in fact a combination of some correct methods proposed long ago and the misleading redefinition of Miller indices.

It is popularly believed that research in traditional fields is low level science, since it is supposed that researchers already have a solid background of fundamentals. However, the reality is that a sceptical and positive attitude toward traditional theories is still relevant in modern research and can lead to valuable changes in methods of interpreting results, encouraging real understanding, reducing mistakes in publications, and eradicating academic misconduct.

\section{Fundamental concepts}

In the conventional definition, Miller indices ( $h k l)$ are three prime integers, i.e., not having a common factor other than +1 or -1 . A specific set of the indices can be obtained from the reciprocals of the intercepts of a specific lattice plane at the lattice axes $\mathbf{a}, \mathbf{b}$, and $\mathbf{c}$ shown by Fig. 1 with the common factor eliminated. It is argued $[1,36]$ wrongly that the prime restriction does not hold when a centered unit cell is chosen. It should be noted that Miller indices define an infinite set of lattice planes having the same orientation but are not specific to any one lattice plane in the family. The indices also define the interplanar spacing of these parallel lattice planes in the lattice. Thus, both the prime property of the definition of Miller indices and the interplanar spacing of lattice planes should not be changed whether the lattice is expressed as primitive or as centered, even though both the chosen axes and the values of the indices can be different when the lattice is expressed differently.

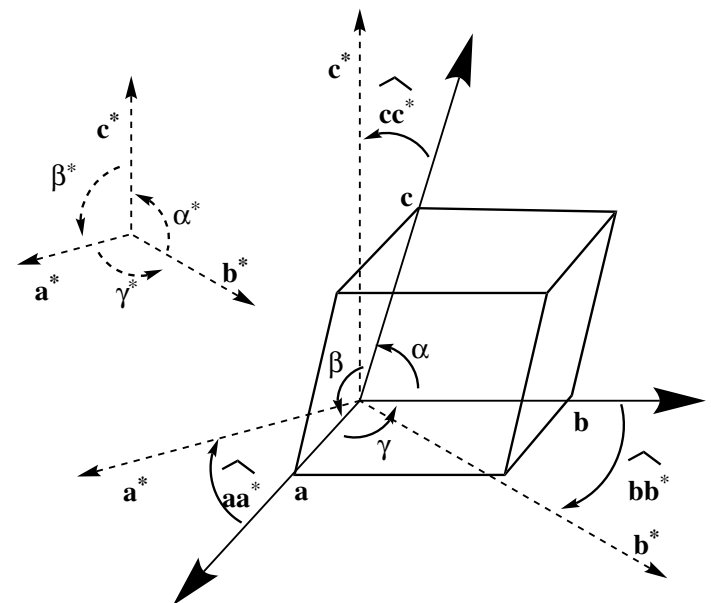

Fig. 1 A unit cell in a lattice with $\mathbf{a}, \mathbf{b}, \mathbf{c}$ as the axes. $\alpha, \beta$, and $\gamma$ are angles between $\mathbf{b}$ and $\mathbf{c}, \mathbf{c}$ and $\mathbf{a}$, and $\mathbf{a}$ and $\mathbf{b}$, respectively. $V$ is the volume of the parallelepiped formed by $\mathbf{a}, \mathbf{b}$, and $\mathbf{c}$. Axes $\mathbf{a}^{*}, \mathbf{b}^{*}, \mathbf{c}^{*}$ and angles $\alpha^{*}, \beta^{*}, \gamma^{*}$ are for the corresponding reciprocal space. $\widehat{\mathbf{a a}}^{*}, \widehat{\mathbf{b b}}$, , and $\widehat{\mathbf{c c}^{*}}$ are angles between $\mathbf{a}$ and $\mathbf{a}^{*}, \mathbf{b}$ and $\mathbf{b}^{*}$, and $\mathbf{c}$ and $c^{*}$, respectively

\subsection{Interplanar spacing between lattice planes}

Miller indices relate to unit vectors in a reciprocal space since both are associated with stacks of parallel planes. Thus, the length of a unit vector $\mathbf{n}$ in a reciprocal space is presented first. The interplanar spacing $d_{h k l}$ for lattice planes with Miller indices ( $h k l)$ in a crystal lattice is equal to the reciprocal of the norm of $\mathbf{n}$ perpendicular to the $(h k l)$ lattice planes [37-39].

$$
\begin{aligned}
|\mathbf{n}|^{2}= & \mathbf{n} \cdot \mathbf{n}=\left(\mathbf{h} \mathbf{a}^{*}+k \mathbf{b}^{*}+l \mathbf{c}^{*}\right) \cdot\left(\mathbf{h} \mathbf{a}^{*}+k \mathbf{b}^{*}+l \mathbf{c}^{*}\right) \\
= & h^{2}\left|\mathbf{a}^{*}\right|^{2}+k^{2}\left|\mathbf{b}^{*}\right|^{2}+I^{2}\left|\mathbf{c}^{*}\right|^{2} \\
& +2 h k\left|\mathbf{a}^{*}\right|\left|\mathbf{b}^{*}\right| \cos \gamma^{*} \\
& +2 h l\left|\mathbf{a}^{*}\right|\left|\mathbf{c}^{*}\right| \cos \beta^{*}+2 k|| \mathbf{b}^{*}|| \mathbf{c}^{*} \mid \cos \alpha^{*} \\
= & h^{2}\left(\frac{|\mathbf{b}||\mathbf{c}| \sin \alpha}{V}\right)^{2}+k^{2}\left(\frac{|\mathbf{c}||\mathbf{a}| \sin \beta}{V}\right)^{2} \\
& +I^{2}\left(\frac{|\mathbf{a}||\mathbf{b}| \sin \gamma}{V}\right)^{2} \\
& +2 h k \frac{|\mathbf{b}||\mathbf{c}| \sin \alpha}{V} \frac{|\mathbf{c}||\mathbf{a}| \sin \beta}{V} \frac{\cos \alpha \cos \beta-\cos \gamma}{\sin \alpha \sin \beta} \\
& +2 h l \frac{|\mathbf{b}||\mathbf{c}| \sin \alpha}{V} \frac{|\mathbf{a}||\mathbf{b}| \sin \gamma}{V} \frac{\cos \alpha \cos \gamma-\cos \beta}{\sin \alpha \sin \gamma} \\
& +2 k l \frac{|\mathbf{c}||\mathbf{a}| \sin \beta}{V} \frac{|\mathbf{a}||\mathbf{b}| \sin \gamma}{V} \frac{\cos \beta \cos \gamma-\cos \alpha}{\sin \beta \sin \gamma}
\end{aligned}
$$

The parameters used in Eq. 1 are indicated in Fig. 1. The formulae for $\cos \alpha^{*}, \cos \beta^{*}$, and $\cos \gamma^{*}$ needed in the derivation are taken from Ref. [39]. The derivation of Eq. 1 is similar to a derivation for a special case for calculating interplanar 
spacing in a rhombohedral lattice [39]. Equation 1 can be replaced alternatively with a more involved derivation as shown by Eq. 2. where $\mathbf{G}^{*}$ is the metric tensor in reciprocal space, the determinant of which equals the square of the volume of the parallelepiped formed by $\mathbf{a}^{*}, \mathbf{b}^{*}, \mathbf{c}^{*}[13,40]$. Both Eqs. 1

$$
\begin{aligned}
& |\mathbf{n}|^{2}=\mathbf{n} \cdot \mathbf{n}^{\top}=\left(\begin{array}{lll}
h & k & l
\end{array}\right)\left(\begin{array}{l}
\mathbf{a}^{*} \\
\mathbf{b}^{*} \\
\mathbf{c}^{*}
\end{array}\right)\left(\begin{array}{lll}
\mathbf{a}^{*} & \mathbf{b}^{*} & \mathbf{c}^{*}
\end{array}\right)\left(\begin{array}{l}
h \\
k \\
l
\end{array}\right) \\
& =\left(\begin{array}{lll}
h & k & l
\end{array}\right) \mathbf{G}^{*}\left(\begin{array}{l}
h \\
k \\
l
\end{array}\right) \\
& =\left(\begin{array}{lll}
h & k & l
\end{array}\right)\left(\begin{array}{ccc}
\left(\frac{|\mathbf{b}||\mathbf{c}| \sin \alpha}{V}\right)^{2} & \frac{|\mathbf{a}||\mathbf{b}||\mathbf{c}|^{2}}{V} \frac{\cos \alpha \cos \beta-\cos \gamma}{V} & \frac{|\mathbf{a}||\mathbf{b}|^{2}|\mathbf{c}|}{V} \frac{\cos \alpha \cos \gamma-\cos \beta}{V} \\
\frac{\left.|\mathbf{a}||\mathbf{b}| \mathbf{c}\right|^{2}}{V} \frac{\cos \alpha \cos \beta-\cos \gamma}{V} & \left(\frac{|\mathbf{c}||\mathbf{a}| \sin \beta}{V}\right)^{2} & \frac{|\mathbf{a}|^{2}|\mathbf{b}||\mathbf{c}|}{V} \frac{\cos \beta \cos \gamma-\cos \alpha}{V} \\
\frac{|\mathbf{a}||\mathbf{b}|^{2}|\mathbf{c}|}{V} \frac{\cos \alpha \cos \gamma-\cos \beta}{V} & \frac{|\mathbf{a}|^{2}|\mathbf{b}||\mathbf{c}|}{V} \frac{\cos \beta \cos \gamma-\cos \alpha}{V} & \left(\frac{|\mathbf{a}||\mathbf{b}| \sin \gamma}{V}\right)^{2}
\end{array}\right)\left(\begin{array}{l}
h \\
k \\
l
\end{array}\right) \\
& =\frac{\left(\begin{array}{lll}
h & k & l
\end{array}\right)\left(\begin{array}{l}
\frac{h}{|\mathbf{a}|^{2}} \sin ^{2} \alpha+\frac{k}{|\mathbf{a}| \mathbf{b} \mid}(\cos \alpha \cos \beta-\cos \gamma)+\frac{1}{|\mathbf{a}| \mathbf{c} \mid}(\cos \alpha \cos \gamma-\cos \beta) \\
\frac{h}{|\mathbf{a}||\mathbf{b}|}(\cos \alpha \cos \beta-\cos \gamma)+\frac{k}{|\mathbf{b}|^{2}} \sin ^{2} \beta+\frac{1}{|\mathbf{b}||\mathbf{c}|}(\cos \beta \cos \gamma-\cos \alpha) \\
\frac{h}{|\mathbf{a}| \mathbf{c} \mid}(\cos \alpha \cos \gamma-\cos \beta)+\frac{k}{|\mathbf{b}||\mathbf{c}|}(\cos \beta \cos \gamma-\cos \alpha)+\frac{1}{|\mathbf{c}|^{2}} \sin ^{2} \gamma
\end{array}\right)}{1-\cos ^{2} \alpha-\cos ^{2} \beta-\cos ^{2} \gamma+2 \cos \alpha \cos \beta \cos \gamma} \\
& =\left\{\frac { h } { | \mathbf { a } | } \left[\frac{h}{|\mathbf{a}|}\left(1-\cos ^{2} \alpha\right)+\frac{l}{|\mathbf{c}|}(\cos \alpha \cos \gamma-\cos \beta)\right.\right. \\
& \left.+\frac{k}{|\mathbf{b}|}(\cos \alpha \cos \beta-\cos \gamma)\right] \\
& +\frac{k}{|\mathbf{b}|}\left[\frac{k}{|\mathbf{b}|}\left(1-\cos ^{2} \beta\right)+\frac{h}{|\mathbf{a}|}(\cos \alpha \cos \beta-\cos \gamma)\right. \\
& \left.+\frac{l}{|\mathbf{c}|}(\cos \beta \cos \gamma-\cos \alpha)\right] \\
& +\frac{l}{|\mathbf{c}|}\left[\frac{1}{|\mathbf{c}|}\left(1-\cos ^{2} \gamma\right)+\frac{k}{|\mathbf{b}|}(\cos \beta \cos \gamma-\cos \alpha)\right. \\
& \left.\left.+\frac{h}{|\mathbf{a}|}(\cos \alpha \cos \gamma-\cos \beta)\right]\right\} /\left|\begin{array}{ccc}
1 & \cos \gamma & \cos \beta \\
\cos \gamma & 1 & \cos \alpha \\
\cos \beta & \cos \alpha & 1
\end{array}\right| \\
& =\left[\frac{h}{|\mathbf{a}|}\left|\begin{array}{ccc}
\frac{h}{|\mathbf{a}|} & \cos \gamma & \cos \beta \\
\frac{k}{|\mathbf{b}|} & 1 & \cos \alpha \\
\frac{1}{|\mathbf{c}|} & \cos \alpha & 1
\end{array}\right|+\frac{k}{|\mathbf{b}|}\left|\begin{array}{ccc}
1 & \frac{h}{|\mathbf{a}|} & \cos \beta \\
\cos \gamma & \frac{k}{|\mathbf{b}|} & \cos \alpha \\
\cos \beta & \frac{1}{|\mathbf{c}|} & 1
\end{array}\right|\right. \\
& \left.+\frac{l}{|\mathbf{c}|} \mid \begin{array}{ccc}
1 & \cos \gamma & \frac{h}{|\mathbf{a}|} \\
\cos \gamma & 1 & \frac{k}{|\mathbf{b}|} \\
\cos \beta & \cos \alpha & \frac{1}{|\mathbf{c}|}
\end{array}\right]|/| \begin{array}{ccc}
1 & \cos \gamma & \cos \beta \\
\cos \gamma & 1 & \cos \alpha \\
\cos \beta & \cos \alpha & 1
\end{array} \mid \\
& =\left[\left(\frac{h \sin \alpha}{|\mathbf{a}|}\right)^{2}+\left(\frac{k \sin \beta}{|\mathbf{b}|}\right)^{2}+\left(\frac{\mid \sin \gamma}{|\mathbf{c}|}\right)^{2}\right. \\
& +2 h k \frac{\cos \alpha \cos \beta-\cos \gamma}{|\mathbf{a}||\mathbf{b}|} \\
& +2 h l \frac{\cos \alpha \cos \gamma-\cos \beta}{|\mathbf{a}||\mathbf{c}|} \\
& \left.+2 k l \frac{\cos \beta \cos \gamma-\cos \alpha}{|\mathbf{b}||\mathbf{c}|}\right] /\left|\begin{array}{ccc}
1 & \cos \gamma & \cos \beta \\
\cos \gamma & 1 & \cos \alpha \\
\cos \beta & \cos \alpha & 1
\end{array}\right|
\end{aligned}
$$


and 2, which are more detailed forms of Eq. 46 in [39], involve some serious algebraic derivations, which cannot be simplified further except by omitting all the detailed derivations, but both equations have their own merits. It should be noted that the omission of some involved derivations from a majority of crystallography monographs does not mean that the omitted materials can be neglected. Readers who have followed such truncated derivations might take it for granted that the omissions are easy and so can be neglected. However, for newcomers to the field a detailed derivation provides an easy way to fill the gap caused by the omissions. For that reason, we have used here detailed schemes that can be followed by a wide range of readers.

Both Eqs. 1 and 2 provide the same result as is obviously required though they take different forms. The equivalence of Eqs. 1 and 2 can be observed with the help of Eq. 3 from which $\cos \widehat{\mathbf{c c}}^{*}$ can also be obtained [39].

$$
\begin{aligned}
& |V|^{2}=[(\mathbf{a} \times \mathbf{b}) \cdot \mathbf{c}]^{2}=\left(|\mathbf{a}||\mathbf{b}||\mathbf{c}| \sin \gamma \cos \widehat{\mathbf{c c}^{*}}\right)^{2} \\
& =\left\|\begin{array}{lll}
c_{x} & c_{y} & c_{z} \\
a_{x} & a_{y} & a_{z} \\
b_{x} & b_{y} & b_{z}
\end{array}\right\|^{2}=\left|\left(\begin{array}{lll}
a_{x} & a_{y} & a_{z} \\
b_{x} & b_{y} & b_{z} \\
c_{x} & c_{y} & c_{z}
\end{array}\right)\left(\begin{array}{lll}
a_{x} & b_{x} & c_{x} \\
a_{y} & b_{y} & c_{y} \\
a_{z} & b_{z} & c_{z}
\end{array}\right)\right| \\
& =\left|\left(\begin{array}{l}
\mathbf{a} \\
\mathbf{b} \\
\mathbf{c}
\end{array}\right)\left(\begin{array}{lll}
\mathbf{a} & \mathbf{b} & \mathbf{c}
\end{array}\right)\right|=\left|\begin{array}{llll}
\mathbf{a} \cdot \mathbf{a} & \mathbf{a} \cdot \mathbf{b} & \mathbf{a} \cdot \mathbf{c} \\
\mathbf{b} \cdot \mathbf{a} & \mathbf{b} \cdot \mathbf{b} & \mathbf{b} \cdot \mathbf{c} \\
\mathbf{c} \cdot \mathbf{a} & \mathbf{c} \cdot \mathbf{b} & \mathbf{c} \cdot \mathbf{c}
\end{array}\right|=|\mathbf{G}| \\
& =(|\mathbf{a}||\mathbf{b}||\mathbf{c}|)^{2}\left|\begin{array}{ccc}
1 & \cos \gamma & \cos \beta \\
\cos \gamma & 1 & \cos \alpha \\
\cos \beta & \cos \alpha & 1
\end{array}\right| \\
& =|\mathbf{a}|^{2}|\mathbf{b}|^{2}|\mathbf{c}|^{2}\left(1-\cos ^{2} \alpha-\cos ^{2} \beta-\cos ^{2} \gamma+2 \cos \alpha \cos \beta \cos \gamma\right)
\end{aligned}
$$

where $a_{x}, a_{y}, a_{z}$ etc. are vector components in the Cartesian system; and $\mathbf{G}$ is the metric tensor in direct space, the determinant of which equals $V^{2}$. It is clear that both Eqs. 1 and 2 are obtained from the same reciprocal method and either can be chosen to develop the method. However, the same result can also be obtained from a number of other different methods, from elementary geometry to vector algebra via Cartesian coordinates [39]. For example, the method via Cartesian coordinates, termed here as the intermediate method, only involves vector algebra and it is less advanced than the advanced reciprocal method exemplified by Eqs. 1 and 2 and thus less complicated. It is obvious that when the same result can be obtained from different methods, it does not follow that the methods are the same. This intermediate method is the most similar to the advanced reciprocal method, but it does not follow that the former is derived from the latter. On the other hand, it is more appropriate to say that the advanced reciprocal method is developed from the intermediate method, or that the concepts in reciprocal space are developed from those in direct space. A third method, described as the elementary geometric method is the simplest and it involves the least amount of advanced mathematics. However, this method is not directly related to the reciprocal method even though the final result is the same.

The method with intermediate Cartesian axes involves vector algebra, but the difficulties involved are primarily to be found with the algebra. The method with elementary geometry involves trigonometry, but the difficulties involved are also concerned with the algebra, though the reciprocal method certainly involves some serious algebra [39]. To obtain the same result by a variety of distinguishable theoretical methods is always valuable and worthwhile particularly when they cannot be straightforwardly obtained from each other. The derivations in Eqs. 1 and 2 are already complex as indeed are the procedures involved in the concepts of reciprocal vectors. The general preference nowadays for the more involved reciprocal method is because its derivation is facilitated by introducing the concepts of reciprocal vectors, and the properties of reciprocal and vector identities. It is simplistic to think that the simplest method of solving a particular problem is to be preferred and other more complicated methods are unnecessary and should be ignored. For significant scientific development, all different methods, however complicated, should be considered as they expand our knowledge of the subject [41].

It should be noted that the symbol || || used in Eq. 3 where the first || stands for determinant and the second | | stands for absolute value of the determinant since the determinant can be positive or negative depending on the directions of the related vectors. Since the determinant of $V^{2}$ can never be negative, a second $\|$ is unnecessary. Thus, the symbol || || used for $\mathbf{n}$ in Ref. [3] should be replaced by || since $\sqrt{\mathbf{n} \cdot \mathbf{n}}$ is positive by definition and independent of the direction of $\mathbf{n}$.

\subsection{The essentials imbedded in the conventional definition of Miller indices}

There are criticisms $[1,3]$ that have been made against the conventional definition of Miller indices involving arguments that the definition has not been associated with an individual lattice plane. Miller indices are related to the interplanar spacings in lattice. The Miller indices for each family of parallel lattice planes must be unique, which is achieved in the conventional definition by requiring them to be co-prime. The reason why Miller indices are not associated with an individual plane is that all lattice nodes are equivalent by translations so the origin can be set on any lattice node. The definition of lattice nodes includes the statement that the environment of every node is the same which needs not be connected by any symmetry operation other than translation. Thus, the environments of nodes at the corners and at the face centers of a $C F$ lattice 
cell are not different since both the points at the corners and at the face centers are lattice nodes. Failure to realize the imbedded principles in the conventional definition of Miller indices leads to the claim that it is impossible to have different environments around two physically distinct but symmetrically equivalent atoms [3]. A detailed discussion on the issue is given in Sect. 2.4.

The individual choices of the axes $\mathbf{a}, \mathbf{b}$, and $\mathbf{c}$ are not relevant when Miller indices are involved, as the crystal is entirely defined by the orientations of the planes. The ratios $h: k: I$ define orientations. Failure to realize this fundamental truth results in the unreasonable criticism [3] of the fundamental principle of translation equivalence in a lattice.

Another advantage of the conventional definition of Miller indices is that the indices must be rational numbers which reflects an important property of lattices, summarized by the law of rational indices. Relative to a coordinate system, all the other planes in a crystal satisfy this law in which a ratio between small, whole numbers is considered to be rational and co-prime when the numbers concerned have no common integral factor greater than one. This feature inherent in the conventional definition of Miller indices ensures that the planes with the same Miller indices in the same lattice are all equivalent. Failure to appreciate this fact has led to mistakes in calculations concerning lattice density and interplanar spacing $[4,5]$ which are discussed in Sects. 3 and 4.

The modified definition [1,3] involves assigning the Miller indices to an individual plane in a family of parallel planes, i.e., by assigning the Miller indices to the plane adjacent to the one passing through the origin, a process which can lead to non-co-prime Miller indices. Although the modification can still uniquely define the indices and has advantages over the conventional definition in some limited cases, the downside is that all the advantages of the conventional definition discussed above are lost. What is more, it can be shown that the modification does not offer any significant advantages except for specific cases, as indicated in Sect. 3.4, when the calculations are actually carried out. Thus, this modification is not likely to become the norm as it was previously proposed as long ago [1] as 1935 but has not become generally accepted in the subsequent 85 years. On the contrary, the conventional definition of Miller indices has stood the test of time.

The fundamental principles imbedded in the conventional definition of Miller indices include the concept that nodes in a lattice are all equivalent and that the lattice planes in a parallel set of a family are all equivalent. Failure to accept the advantages inherent within the conventional definition leads to the redefinition of the conventional definition of Miller indices. In fact, the equation (Eq. 4) for lattice planes rather than Miller indices should be used if an individual lattice plane needs to be referred to.

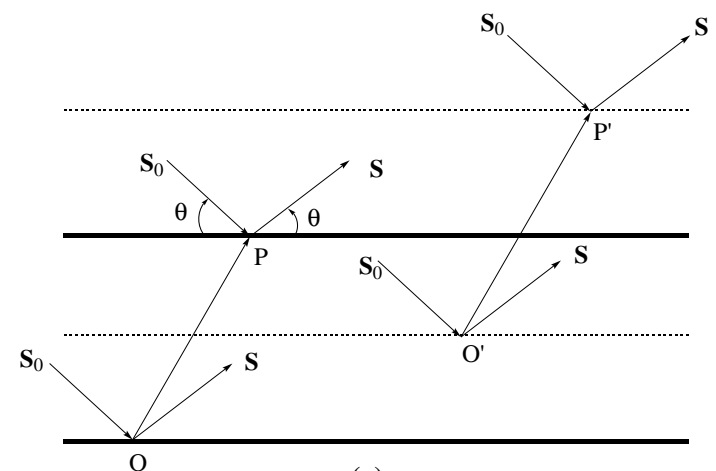

(a)

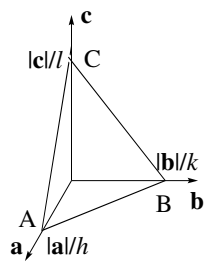

(b)

Fig. 2 a Bold horizontal lines indicate lattice planes passing though points $\mathrm{O}$ and $\mathrm{P}$ for a primitive lattice. A non-primitive lattice can be constructed by inserting planes symmetrically placed between adjacent planes in a primitive lattice. Dashed horizontal lines indicate these inserted planes passing $\mathrm{P}^{\prime}$ and $\mathrm{O}^{\prime}$. $\mathbf{S}_{0}$ and $\mathbf{S}$ are the unit vectors for the incident and reflected beams, respectively. OP and $\mathbf{O}^{\prime} \mathbf{P}^{\prime}$ are vectors associated with points on different planes. $\theta$ is the Bragg angle characterizing the reflection from the planes. $\mathbf{b}$ A plane characterized by Miller indices $(h k l)$

$h x+k y+k z=n$

where $(h k l)$ are Miller indices, $x, y, z$ are the coordinates of a lattice point, and $n$ is an integer for primitive lattice or rational number for non-primitive lattice. It was insisted $[1,3]$ that assigning (111) to Miller indices in the conventional definition for the I-centered cubic lattice was wrong because $n$ in Eq. 4 must be an integer, and assigning (111) to Miller indices contradicted the fact that $n=1$ represented the plane closest to the origin [for example the plane passing the node at $(x, y, z)=(1 / 2,1 / 2,-1 / 2)]$. However, assigning (111) as the Miller indices of this plane is not wrong. For a primitive lattice, $x, y$, and $z$ are integers and thus $n$ must be integral and is equal to 1 for the plane closest to the origin in a family of planes. For a nonprimitive lattice, $x, y$, and $z$ can be fractionals and thus $n$ can also be fractional and indeed $n=1$ may not represent the plane closest to the one passing through the origin. In the conventional definition, Miller indices do not refer to an individual lattice plane though they could be obtained from one particular plane.

\subsection{Diffraction indices and Miller indices}

Diffraction indices are defined by Laue equations 
$\mathbf{a} \cdot\left(\mathbf{S}-\mathbf{S}_{0}\right)=h \lambda$

$\mathbf{b} \cdot\left(\mathbf{S}-\mathbf{S}_{0}\right)=k \lambda$

$\mathbf{c} \cdot\left(\mathbf{S}-\mathbf{S}_{0}\right)=\lambda$

where $\lambda$ is the wavelength of the $\mathrm{X}$-rays and $\mathbf{S}$ and $\mathbf{S}_{0}$ have been shown in Fig. 2. The $h k l$ in Eqs. 5-7 are diffraction indices. The diffraction (Laue) indices can be obtained by multiplying the Miller indices with a common factor and are written without parentheses to distinguish them from the latter. Both sets of indices are consistent with Eq. 4 for lattice planes. Thus, interplanar distances are imbedded in the calculation of structure factors $F_{h k l}$ shown by Eq. 8

$F_{h k l}=\sum_{i} f_{i} e^{2 \pi j\left(h x_{i}+k y_{i}+k z_{i}\right)}$

The structure factor for the diffraction indices $h k l$ is calculated by summing the contributions in a unit cell of every atom i at position $x_{i}, y_{i}, z_{i}$ with scattering factor $f_{i}$. The interdependence of diffraction indices, interplanar distances, and structure factors is imbedded in Eq. 8. Using Eqs. 5-7 for vector OP in Fig. 2a,

$$
\begin{aligned}
\mathbf{O P} \cdot\left(\frac{\mathbf{S}}{\lambda}-\frac{\mathbf{S}_{0}}{\lambda}\right) & =\left(x_{P} \mathbf{a}+y_{p} \mathbf{b}+z_{P} \mathbf{c}\right) \cdot\left(\frac{\mathbf{S}}{\lambda}-\frac{\mathbf{S}_{0}}{\lambda}\right) \\
& =h x_{P}+k y_{P}+z_{P} \\
& =\left(x_{P} \mathbf{a}+y_{P} \mathbf{b}+z_{P} \mathbf{c}\right) \cdot\left(h \mathbf{a}^{*}+k \mathbf{b}^{*}+l \mathbf{c}^{*}\right) \\
& =\left(x_{P} \mathbf{a}+y_{P} \mathbf{b}+z_{P} \mathbf{c}\right) \cdot \mathbf{r}
\end{aligned}
$$

When the diffraction indices $h k l$ are the same as the Miller indices $(h k l), \mathbf{r}$ in Eq. 9 becomes the unit vector $\mathbf{n}$.

$$
\frac{\mathbf{S}}{\lambda}-\frac{\mathbf{S}_{0}}{\lambda}=\mathbf{n}
$$

From Eq. 10 and Ewald's sphere, [39], we obtain

$\left|\frac{\mathbf{S}}{\lambda}-\frac{\mathbf{S}_{0}}{\lambda}\right|=\frac{1}{d_{h k l}}=\frac{2 \sin \theta}{\lambda}$

An aplanatic plane for a particular diffraction is a reflection plane since all the diffracted beams travel the same length of optical path from the plane [21, 42]. It can be proved that the planes with Miller indices can be reflection planes for a particular diffraction. Equation 9 will not be affected when $P$ is changed to any lattice point on the plane passing through $\mathrm{P}$ in Fig. 2a, consistent with the conventional definition of Miller indices. In fact, OP can represent any vector between planes with the same interplanar spacing characterized by Miller indices (hkl). For example, Eq. 9 will not be affected if $\mathbf{O P}$ is changed to $\mathbf{O}^{\prime} \mathbf{P}^{\prime}$ in Fig. 2a, i.e., Eq. 9 characterizes the property of a family of planes with the same Miller indices, not an individual plane. This is another reason why Miller indices cannot be associated with a particular plane. From Fig. $2 b$ and Eqs. 5-10, we obtain

$\left(\frac{\mathbf{b}}{k}-\frac{\mathbf{a}}{h}\right) \cdot\left(\frac{\mathbf{S}}{\lambda}-\frac{\mathbf{S}_{0}}{\lambda}\right)=\mathbf{A B} \cdot \mathbf{n}=0$

$\left(\frac{\mathbf{c}}{l}-\frac{\mathbf{a}}{h}\right) \cdot\left(\frac{\mathbf{s}}{\lambda}-\frac{\mathbf{S}_{0}}{\lambda}\right)=\mathbf{A C} \cdot \mathbf{n}=0$

Equations 12 and 13 show that the plane $A B C$ (Fig. $2 b$ ) is really a reflection plane, i.e., the diffractions characterized by Eqs. 5-7 are essentially reflections from planes with Miller indices $(h k l)$.

If the diffraction indices $h k l$ are for first-order diffraction, then the second-order indices are $(2 h)(2 k)(2 l)$. When a plane is symmetrically inserted between the two adjacent planes, second-order diffraction is changed to first-order diffraction $[43,44]$. This effect is analogous to the change in interplanar spacing when a primitive lattice is changed into a non-primitive lattice by inserting planes (Fig. 2a) and also shows that diffraction and interplanar spacing are closely related. The interplanar distances for non-primitive lattices are used in [44] to account for the fact that both 222 and 111 may be allowed for diffraction indices while the correct nomenclature for Miller indices is (111) but not (222) as claimed in [3].

\subsection{The environment of every node in a lattice is the same}

When considering whether a common factor is allowed or not, we admire the precision and relevance of the conventional definitions of diffraction and Miller indices. Another problem associated with the failure of seeing the equivalence of nodes imbedded in the conventional definition of Miller indices is the development of incorrect statements such as "symmetry-related sites are identical" or "it is obviously simply impossible to have different environments around two physically distinct but symmetrically equivalent atoms" [3].

\subsubsection{Equivalent and identical positions}

The adjectives equivalent and identical have different meanings when applied to positions. As shown in Fig. 3, symmetry-related positions are not always identical but do offer some kind of equivalence. For example, the environments of two chiral positions related by an $S_{2}$ axis 


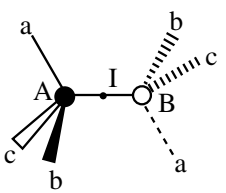

(a) Sites A and B are equivalent but distinguishable symmetrically

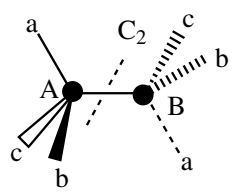

(b) Sites A and B are identical and indistinguishable symmetrically in molecule

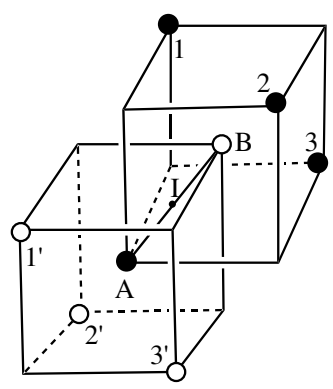

(c) Sites A and B in diamond

Fig. 3 The two positions $A$ and $B$ are related by an inversion center $\mathrm{I}$ in $\mathbf{a}$, by $\mathrm{C}_{2}$ operation in $\mathbf{b}$, and by both the inversion center $\mathrm{I}$ and the $C_{2}$ axis in $\mathbf{c}$. The symbols $A$ and $B$ used in $\mathbf{b}$ do not refer to different types but are an indication of different positions. There is an $\mathrm{S}_{6}$ axis along $A B$ in $c$ which is along the common diagonals of the two interlocked cubic units or the line connecting the centers of $\Delta 123$ and $\Delta 1^{\prime} 2^{\prime} 3^{\prime}$

perpendicular to $A B$ or an inversion center I are not identical and therefore distinguishable (Fig. 3a). Positions $A$ and $B$ associated by the $C_{2}$ axis in Fig. $3 b$ in an isolated molecule are identical and therefore indistinguishable since the molecule can rotate freely and thus the environments of the two sites are the same. Positions $A$ and $B$ in Fig. $3 c$, as an isolated structure unit in the gas phase, are identical with respect to both the $C_{2}$ axis perpendicular to $A B$ and an inversion center but their environments are not identical since the chirality is different [12]. When the positions are considered to be contained within a crystal, environments of positions A and B in Fig. 3 are apparently not identical since the unit cannot then rotate freely and the two positions are not associated with a translation operation from $A$ to $B$. Lattice nodes must have the same environment with respect to the symmetry of translation. If $A$ in Fig. 3 is chosen as a lattice node, then B cannot be a lattice node since the environments of the two sites are different. Rotating the whole crystal with structure units such as those symmetry operations shown by Fig. 3 cannot change the anisotropic property of the crystal.

Classification is determined by criterion. Even though sites $A$ and $B$ can be occupied by the same kind of atoms or with some kind of equivalence, they can either be classified as the same type by the criterion of specific symmetry operations or classified into different types by the criterion of environment.

\subsubsection{Asymmetric unit, unit cell, and environment}

The environments around positions $A$ and $B$ in unit cells in Fig. 4 are different, and this can be addressed from lattice theory. A unit cell with one lattice node is primitive or simple if the axes are chosen in such a way that all the lattice nodes have integral coordinates. A unit cell is centered or multiple if there are translations with non-integral coordinates and then the unit cell contains several lattice nodes. The space group for the diamond crystal is $O_{h}^{7}-F d \overline{3} m$ (No. 227). The diamond unit cell in this space group can be constructed by providing only one carbon atom with coordinates $(0,0,0)$ as all the other atoms in the conventional face-centered cubic cell $c F$ for the diamond crystal (Fig. 4a) are then determined by symmetry [26]. But that fact does not mean that the unit cell for diamond only contains one atom. There are two carbon atoms with different environments in the primitive unit cell for the diamond crystal as shown by Figs. 3c and 4b. From symmetry equivalence, the eight carbon atoms in the conventional cubic cell of diamond belong to the same kind since they are related by symmetry operations. However, the classification of position type depends on the criterion. Sites $A$ and $B$ in Fig. $3 a$ are not identical since their chiral sites $R$ and $S$ are different. The two carbon atoms in the primitive unit cell of diamond have some kind of difference since the environments of the two positions are different (Fig. 3c). Thus, the symbols $A$ and $B$ can be introduced to differentiate positions.

There are doubts about the statement that "The environment around a carbon $A$ is different from that around a carbon $B^{\prime \prime}$ in the structure of diamond. The reasoning that "The structure of diamond contains only one atom in the asymmetric unit; it is obviously simply impossible to have different environments around two physically distinct but symmetrically equivalent atoms" [3] confuses the concepts of asymmetric unit and environmental equivalence. A particular symmetry operation relating positions within a set of Wyckoff sites can indeed allow fewer atoms in an asymmetric unit, as for example two atoms of a $\mathrm{ZnS}$ unit in zinc blende but a single carbon atom in diamond when there are symmetry operations relating site $A$ to site $B$ as shown in Fig. 3c.

\subsubsection{Wyckoff site, lattice node, and environment}

A symmetry operation cannot ensure the equivalence of the positions in Fig. 4 in the directions that are required by a lattice node in a crystal if the positions in the Wyckoff site have not all been converted into lattice nodes by the operation. For example, the number of atoms in the primitive cell for diamond is still two while only one in the asymmetric unit. As shown by their characters, symmetry 


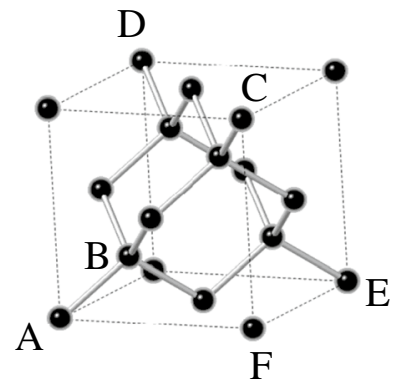

(a)

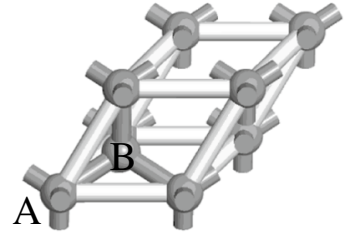

(b)

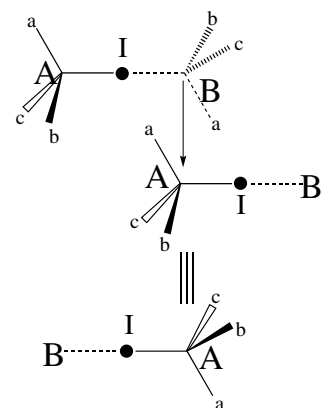

(c)

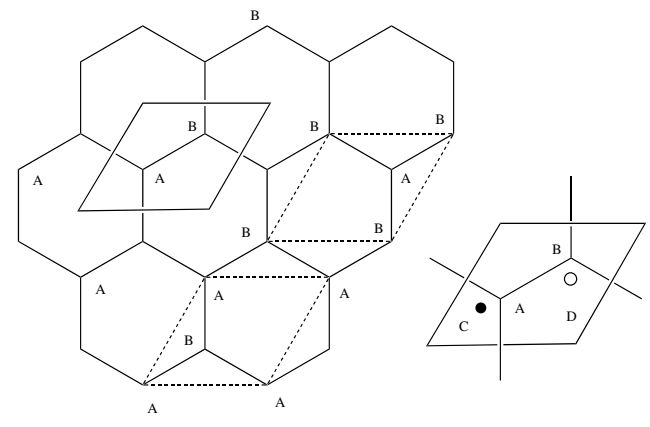

(d)

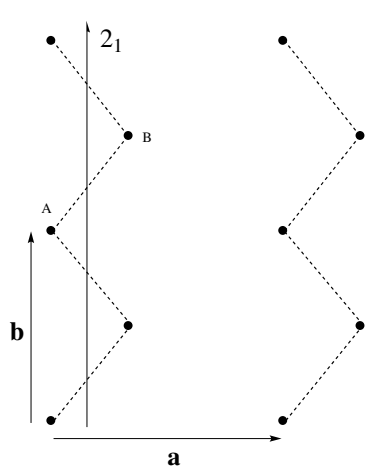

(e)
Fig. 4 The conventional non-primitive face-centered cubic cell (a) and the primitive rhombohedral unit cell (b) for diamond. The translation by the vector connecting carbon atoms $\mathrm{A}$ and $\mathrm{B}$ cannot shift the crystal into itself even though the two atoms are related by inverse centers, diamond glides $d$, and screw rotations $4_{1}$. Two physically distinct sites $A$ and $B$ in a unit cell with different environments in (c) are related by a center of symmetry. If a chiral carbon atom is based on position $A$, then position $B$ will be occupied by a carbon atom of opposite chirality. So, the environments of the sites are different. When $A$ is translated to $B$, the environment of $B$ is

operations in a group behave differently in a symmetry species. Different symmetry operations demonstrate different kinds of equivalence. If symmetry-related sites are identical in every aspect, then only one symmetry operation would be sufficient. In fact, both the number of symmetry operations and their type are significant. The translation operation in crystals provides information not expressed by other types of symmetry operations. Lattice nodes are identical with respect to translation symmetry, i.e., the nodes at the face centers and at the corners in the unit cell for a $C F$ lattice are indistinguishable since their environments are the same with respect to translations. If the environments of two sites are different with respect to translation such as those of the two carbon atoms in the unit cell of diamond, the two sites cannot both be lattice nodes at the same time even when they are related by another type of symmetry.

An isolated molecule can rotate freely where the environments of two different achiral sites can be identical transformed from that indicated by dashed lines to that below indicated by solid lines. Thus, the equivalence from a particular symmetry operation cannot ensure the equivalence of environments. In (d) two physically distinct sites A and B with different environments in graphite are related by sixfold rotation operation $C_{6}$ and an inversion center at the middle of bond A-B. C and D with their own environments are two different sites randomly chosen. The environments of $A, B, C$ and $D$ are all different. $\ln (\mathbf{e})$ points $A$ and $B$ with different environments are related by a 2, screw axis containing a $\mathbf{b} / 2$ translation

if the sites are related by a specific symmetry operation since direction is unimportant. But such a molecule cannot rotate freely in a crystal where direction is crucially important. In a lattice, identical environments can only be ratified by a translation operation rather than other symmetry operations which only relate Wyckoff positions. This is confirmed in Fig. 4 where a particular symmetry operation other than translation cannot provide an identical environment in all directions. That is why Wyckoff sites and lattice nodes are different concepts in crystallography. Wyckoff sites define equivalent positions established by symmetry operations while lattice nodes relate to environmental equivalence from translation operations.

The property of a lattice ensures the spatial equivalence of any lattice node. A lattice must be shifted to itself by a translation with a vector connecting any two lattice nodes. As can be seen from Fig. $4 a$ or $4 b$, the vector connecting carbon atoms $A$ and $B$ in diamond cannot shift the crystal to itself. Thus, the environments around atoms $A$ and $B$ are 
different. That is why two sets of coordinates $(0,0,0)$ and $(1 / 4,1 / 4,1 / 4)$ for the eight positions of Wyckoff site $8 \mathrm{a}$ for space group No. 227 in The International Tables for Crystallography [45] are cited along with the four lattice nodes at $000,0 \frac{1}{2} \frac{1}{2}, \frac{1}{2} 0 \frac{1}{2}$, and $\frac{1}{2} \frac{1}{2} 0$. Spatial equivalence is characterized by translation operations rather than other types of symmetry operation in crystal. Carbon atoms in positions $\mathrm{A}$ and $B$ in diamond are only equivalent when considering the site symmetry of $O_{h}^{7}-F d \overline{3} m$ or diamond glide planes $d$ and screw rotations $4{ }_{1}$ but the environments of the two atoms are shown to be not identical by translation operations such as that involving the vector from atom $A$ to $B$ in the diamond crystal.

The difference in environment between the two positions $A$ and $B$ in diamond is not readily apparent unless the two sites are occupied by different chemical species. The distinction between positions can be manifested by replacing the carbon atom at position- $\mathrm{A}$ with an $\mathrm{S}^{2-}$ ion and the carbon atom at position-B with a $\mathrm{Zn}^{2+}$ ion to form zinc blende ( $\mathrm{ZnS})$. The equivalence of the two positions in diamond from symmetry operations such as inversion at $(1 / 8,1 / 8,1 / 8)$, the 4 screw rotation, and the diamond glide is removed in zinc blende and thus the space group is lowered to $T_{d}^{2}-F \overline{4} 3 m$ (No. 216). The eight positions of 8a site in space group No. 227 have been changed to those of the two sites $4 \mathrm{a}$ and $4 \mathrm{c}$ or $4 \mathrm{~d}$ in space group No. 216.

The $\mathrm{Zn}^{2+}$ and $\mathrm{S}^{2-}$ ions in zinc blende are two physically distinct identities. It might be thought that their environments would have been the same if the chemical differences were excluded. Diamond is just such a case since there is no chemical difference between sites $A$ and $B$. Sites $A$ and $B$ are indeed equivalent since they are related by symmetry operations. But the fact that two sites are equivalent via particular symmetry operations does not imply that their environments are also the same. The environments at points $A$ and $B$ in Fig. 4a are apparently different. Equivalent sets of points for B, C, or D in Fig. $4 d$ can be generated by using a vectors of any translation between points from position set $A$, but the environments of the positions between the sets can be different. If a set of simple cubic lattice positions are translated to the body centers, then the two sets of positions together form a $\mathrm{cl}$ lattice. Only in such a case can the environment of every position be the same. Lattice nodes should not be confused with Wyckoff sites. It is a basic property of a lattice that the environment of each node must be the same. In a crystal, the environment can be judged by the property of lattice nodes. The carbon atoms at positions A and B of the Wyckoff site 8a in diamond cannot both be lattice nodes. Although both positions are occupied by the same chemical element in diamond and are associated by symmetry operations, the different environments of these two positions are apparent from the properties of a lattice.

Different chemical elements can selectively occupy sites with minor differences such as that forming the structure of diamond or zinc blende. The difference in environment might be one of the reasons, together with factors such as radius ratios, and electrostatic forces, for the type of crystal that is formed. The ability of inorganic elements or ions to differentiate and occupy different sites led us to propose previously that the nature of inorganic ions, specifically their size and charge, can have a decisive effect in determining the type of crystal structure finally adopted by an inorganic compound. For example, there are cubic and hexagonal blocks in hexagonal barium ferrite. Since $\mathrm{Al}^{3+}$ presented in cubic spinel implies using $\mathrm{Al}^{3+}$ might encourage the formation of cubic structure, we have used $\mathrm{Fe}^{2+}$ and $\mathrm{Al}^{3+}$ to encourage the formation of cubic block in the synthesis of the ferrite from the fact that $\mathrm{Fe}_{3} \mathrm{O}_{4}$ and $\mathrm{MgAl}_{2}$ $\mathrm{O}_{4}$ form a cubic lattice [28]. That is, there are some controls that can be used to direct the final crystal structure which involve altering reaction mechanisms by choosing appropriate inorganic ions [46].

Why do most ionic inorganic materials form crystals instead of amorphous substances? If there is a small preference for different chemical species to occupy different sites, then periodicity will magnify this preference. If there is no difference between sites $A$ and $B$, depending on an entropy or an enthalpy-controlled process, two distinct chemical species could theoretically be randomly distributed. For example, there are four sets of $c P$ nodes in the $c F$ lattice. The environment of each node among the four sets is the same; thus, the chemically distinct $\mathrm{Cu}$ and $\mathrm{Au}$ can be randomly distributed among the nodes to form an alloy of $\mathrm{Cu}_{3} \mathrm{Au}$ with space group $\mathrm{O}_{h}^{5}-\mathrm{Fm} \overline{3} m$ (No. 225). It is also true that the nodes in the sets can be periodically occupied by distinct chemical species which therefore provide different environments for nodes between the sets. Although the four sets of simple cubic lattice nodes in the $c F$ lattice are all the same, the $\mathrm{Cu}$ atoms in $\mathrm{Cu}_{3} \mathrm{Au}$ can occupy one set and the Au atoms occupy the other three sets in a periodical instead of a random way to form an alloy of $\mathrm{Cu}_{3} \mathrm{Au}$ with space group $O_{h}^{1}-P m \overline{3} m$ (No. 221). Similarly, in $\mathrm{CsCl}$, two sets of nodes in the $\mathrm{Cl}$ lattice are occupied by $\mathrm{Cs}^{+}$and $\mathrm{Cl}^{-}$in an ordered periodic manner. A variety of different chemical or physical effects can similarly convert nodes into different environments. For example, the Jahn-Teller effect can reduce the energy of $\mathrm{Cu}_{3} \mathrm{Au}$ which lowers the symmetry of the lattice from cubic $O_{h}^{5}-F m \overline{3} m$ to the orthorhombic $O_{h}^{1}-P m \overline{3} m$ species. Another example is 


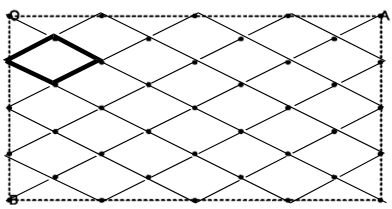

(a)

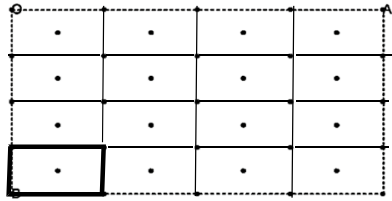

(c)

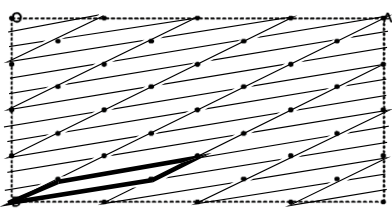

(b)

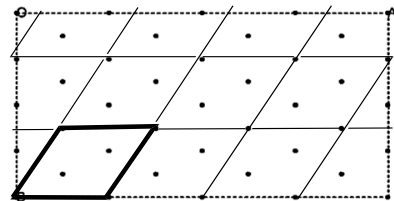

(d)
Fig. 5 The same centered rectangular planar lattice is expressed via a conventional (a) and non-conventional (b) primitive lattice and with conventional (c) and non-conventional (d) multiple lattices. The bold lines indicate the unit cell for the specific lattice

provided by polythene which undergoes Peierls distortion from a structure with equal $\mathrm{C}-\mathrm{C}$ bond lengths to another with distinct bond lengths for the double and the single bonds [47].

\subsubsection{Bravais and non-conventional lattices, and environment}

Although the structure of diamond can be regarded as be composed by two interlocked $c F$ lattices with a total of eight lattice nodes, it is in fact a single $c F$ lattice with only four lattice nodes. This claim is reasonable even though there have been opposing views [3]. There are only fourteen Bravais lattices none of which have more than four lattice nodes in the unit cell. These are conventional lattices but there are an infinite number of non-conventional ones such as those indicated in Fig. 5 all of which represent the same lattice. As all lattice nodes are equivalent, not all the eight carbon atoms in the conventional cubic cell for diamond can be positioned on lattice nodes. The two interpenetrating lattices with a total of eight points in a diamond unit cell cannot together constitute a Bravais lattice with each point as a lattice node but can be reduced to a Bravais lattice with no more than four nodes in its unit cell, which is an indication that the environments of carbon atoms $A$ and $B$ in diamond are not the same even though they are related by some symmetry operations.

There are four lattice points or eight carbon atoms in the conventional face-centered cubic cell for the diamond crystal as shown in Fig. 4a. There is one lattice node or two carbon atoms in its primitive rhombohedral unit cell shown by Fig. $4 \mathrm{~b}$, and the non-conventional I-centered rhombohedral cell by axes AC, AD, and AE (Fig. 4a) with eight lattice points and sixteen carbon atoms is also possible for diamond. ${ }^{1}$ All these different descriptions refer to exactly the same lattice. But it does not mean that there is a Bravais lattice containing eight lattice nodes in its cell. The conventional lattice adopted by the diamond crystal is the face-centered cubic lattice, one of the fourteen Bravais lattices. A unit cell for a Bravais lattice cannot have eight lattice nodes. The mistakes concerning the calculation of interplanar spacing arose $[4,5]$ because both sites $A$ and $B$ in diamond were wrongly taken as lattice nodes.

It can be inferred from Fig. 5 that the spacing for a family of parallel lattice planes cannot be changed by defining the lattice differently and all the nodes have the same environment no matter how the lattice is described. However, the statement that "the structure of diamond contains only one atom in its asymmetric unit" [3] does not guarantee that all the carbon atoms in diamond have the same environment relative to translation.

\subsection{The common usage of the term "diamond lattice"}

There is some controversy [48] about the commonly used [49-55] term of "diamond lattice," such as "the structure of diamond is the prototype of the invariant lattice complex $D$, which negates the ... statement ... :'Put it in other words, diamond is a face-centered lattice, there cannot be two lattices in a face-centered diamond lattice, though it could be said that there were two interpenetrating facecentered cubic lattices'. There exists no such a thing like

\footnotetext{
1 The number of nodes can be more than four in a non-conventional unit cell, but the volume is also bigger than its conventional counterpart. The volume of the non-conventional $l$-centered rhombohedral cell in Fig. 4a is
}

$(\mathbf{A C} \times \mathbf{A D}) \cdot \mathbf{A E}=\left(\sqrt{2}|\mathbf{A F}| \cdot \frac{\sqrt{3}}{2} \sqrt{2}|\mathbf{A F}|\right) \cdot \frac{2}{3} \sqrt{3}|\mathbf{A F}|=2|\mathbf{A F}|^{3}$

or

$\left|\begin{array}{lll}1 & 1 & 0 \\ 0 & 1 & 1 \\ 1 & 0 & 1\end{array}\right||\mathbf{A F}|^{3}=2|\mathbf{A F}|^{3}$

obtained from Eq. 3 with $\alpha=\beta=\gamma=\pi / 3$ and the formula for cos

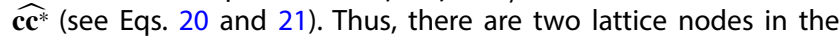
non-conventional 1 -centered rhombohedral cell for a primitive cubic lattice and there are eight lattice nodes in the non-conventional I-centered rhombohedral cell for a face-centered cubic lattice. The eight lattice nodes within the cell are distributed at the eight vertices (which together only contribute one lattice node to the cell), the body center (contributing 1 node), the twelve edgecenters (contributing 3 nodes), and six face-centers (contributing 3 ) of the I-centered rhombohedral cell. There are still eight more carbon atoms at other sites within this cell for the diamond. For closepacking of equal spheres, the vertices of this non-conventional cell are located at every other layer in the sequence $A B C A B C A$ [26]. 
a 'diamond lattice'" given in Ref. [3]. The quotation above uses the term "lattice complex D" to negate the statement that the lattice of diamond is not a new lattice but belongs to one of the 14 Bravais lattices. In fact, "lattice complex $D^{\prime \prime}$ exemplified by diamond is not really a new lattice but is one of the 14 Bravias lattices. "Lattice complex D" and "diamond lattice" are different ways of describing the same thing though the latter is more common. Although the two interpenetrating $F$ lattices in diamond form the composite lattice $D$, the structure of diamond can be described interchangeably by the terms "lattice complex $\mathrm{D}$ " and non-symmorphic "diamond lattice" [55], and it should be pointed out that this latter term has been conveniently used for other structures such as $\mathrm{MgCu}_{2}$ [51] and $\mathrm{MgAl}_{2} \mathrm{O}_{4}$ where $\mathrm{Mg}$ occupies the positions in the "diamond lattice" [49]. In fact, the concepts of lattice and crystal structure cannot be isolated from each other and "lattice" has a broader meaning in crystallography as it is regularly used in terms such as lattice energy, lattice dynamics, and rock salt lattice. It is contradictory to suggest that the terms "lattice complex D" and CsCl lattice can be used but "diamond lattice" is not allowed [3]. "Diamond lattice" is one of the fourteen Bravais lattices with four lattice nodes [39] and each node represents the structure unit specific in diamond. The lattice complex $\mathrm{D}$, a particular lattice form adopted by diamond, is just one among all forms of the $C F$ crystals. For example, the forms of the Bravais $C F$ lattice expressed by diamond and spinel $\mathrm{MgAl}_{2} \mathrm{O}_{4}$ are different even though the two crystals belong to the same space group. An individual lattice can be described in several different ways such as primitive or non-primitive, conventional or non-conventional, and pseudo-lattice such as "lattice complex D" or "diamond lattice". Thus, it is reasonable to use the word "diamond" to define a "lattice" implying that the $C F$ lattice expressed by diamond is in a different form from that expressed by spinel $\mathrm{MgAl}_{2} \mathrm{O}_{4}$, etc. It should be noted that an infinite number of non-conventional and pseudo lattices can be generated from a given lattice. In Fig. 5, the same lattice is expressed in different forms of conventional and non-conventional lattices. The interplanar spacing discussed in [39] applies to both conventional and non-conventional lattices.

\subsection{A summary of Sect. 2}

The very basic, simple, and essential concepts contained in older classical textbooks are very valuable as they often provide a consistent, effective, and critical basis for reviewing of the materials already published [18, 22]. Patience is necessary in reading a textbook or an article from the beginning to the end in order to get every detail correct and obtain real understanding. The conventional definition of Miller indices is more meaningful than it looks at first sight. The fact that the environments of all lattice nodes in a lattice are the same is imbedded in the definition which manifests the fact that the interplanar spacing is not changed whether the lattice is expressed in primitive, non-primitive, and non-conventional lattice forms. It is simplistic to state that the conventional definition of Miller indices is wrong $[1,3]$. Failure to realize the essentials behind the definition has led to mistakes $[4,5]$ discussed in the next two sections. Problems in publications [1-5] are often caused by incorrect understanding of the concepts. Basic principles are still valuable and relevant in serious modern day research. Remarkable results are often published by today's research community, but these are usually based on fundamental theories developed long ago even though this may not be realized at the time. By contrast, it is unfortunate that if a deep understanding is gained from expanding basic principles, the work can be considered as too fundamental to be publishable. We hope that the discussions here can increase the popularity of working in areas of fundamental theory and its applications and attract the attention of researchers to increase their ability to solve new problems with fundamental principles.

\section{Lattice node density in lattice and crystal planes}

In an article discussing lattice node density in lattice planes Fan [4] claimed "new conclusions" derived from a "novel" method of calculating node density in lattice and crystal planes. However, these conclusions were improperly derived by generalizing the results of calculations on specially located atoms in a crystal and by confusing the concepts of lattice nodes and atoms in a crystal. In this section, we discuss our reasons for criticizing these comments and then present a corrected version of the theory applied to non-primitive lattices and crystals. In Sect. 3.4, an important issue concerning the relative lattice node density between planes with different Miller indices is discussed. The method demonstrates that any unit cell, whatever its shape or orientation, contains all the necessary information about the lattice. 


\subsection{All lattice planes in a parallel set with the same Miller indices are equivalent}

All lattice planes in a parallel set with the same Miller indices are equivalent which means every property including node density is the same for each plane. This fundamental principle is imbedded in the conventional definition of Miller indices and disregarding it in recent publications [1-5] has led to mistakes.

The density of lattice nodes in a lattice plane is defined as the number of lattice points per unit area on that plane. It was claimed unjustifiably in [4] that the densities of lattice nodes for a set of parallel lattice planes could be different in non-primitive lattice. This is not true. All the parallel planes in a family must be equivalent in a lattice, a fact consistent with the conventional definition of Miller indices. Thus, the lattice node density in every lattice plane with the same Miller indices $(h k l)$ are identical. The lattice node density for a lattice plane cannot be changed if the lattice representation is changed from primitive to nonprimitive, i.e., lattice node density is a physical property of the crystal and so cannot depend on an arbitrary choice of basis vectors.

In one such paper [4] concerned with lattice node density in lattice planes, it was stated that in order to evaluate node density it was first necessary to define a number [defined as a position-duplication $(P D)$ number] which is a function of $m / q$ where $m$ and $q$ are prime numbers and $m<q$ defined in Eq. 14.

$h x+k y+k z=s+\frac{m}{q}$

where $s$ is an integer. It should be noted that Eq. 14 for a non-primitive lattice is obtained from Eq. 4 by replacing $n$ with $s+m / q$. Fan did not differentiate between the positions of lattice nodes and atoms in crystals and indeed used $\mathrm{m} / \mathrm{q}$ to characterize both. In fact in a crystal, $\mathrm{m} / \mathrm{q}$ can be replaced by a real number instead of a rational number. Fan's conclusion that planar lattice node density can be different in parallel planes within non-primitive lattices was obtained by generalizing from a set of specific calculations but only by confusing the concepts of lattice nodes and atoms in a crystal. We have briefly mentioned previously $[22,39]$ that such generalizations are not justified.

Let us take the face-centered cubic lattice shown in Fig. 6 for example. The open circles used instead of points in Fig. 6 serve as an intuitive way to calculate the number

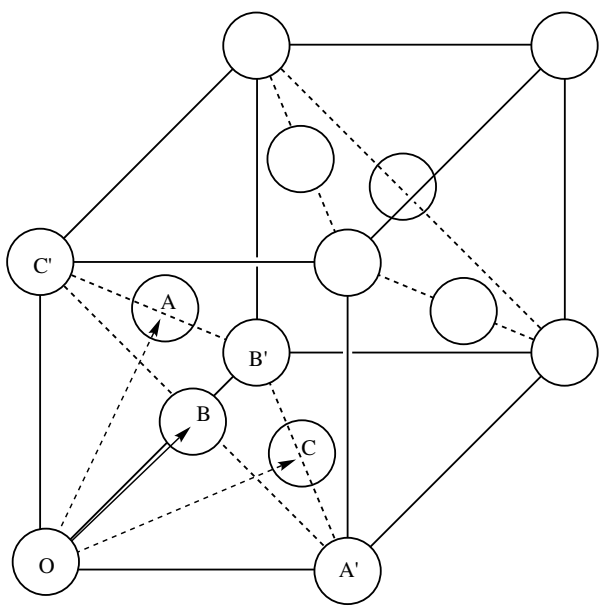

Fig. 6 A face-centered cubic cell. A primitive lattice is based on axes $\mathbf{O A}, \mathbf{O B}$, and $\mathbf{O C}$. A conventional non-primitive lattice is based on axes $\mathbf{O} \mathbf{A}^{\prime}, \mathbf{O} \mathbf{B}^{\prime}$, and $\mathbf{O} \mathbf{C}^{\prime}$. Open circles represent lattice nodes. The node density for the plane with Miller indices (111) is $\rho\left(S_{111}, C F\right)=\frac{1 / 2}{S_{A B C}}=\frac{2}{S_{A^{\prime} B^{\prime} C^{\prime}}}=\frac{4}{\sqrt{3}\left|\mathbf{O A}^{\prime}\right|^{2}}$

of nodes in a certain area. ${ }^{2}$ Each open circle, indicating a lattice node, at $A, B$, or $C$ contributes $1 / 6$ or $\pi / 3$ out of $2 \pi$ to triangle $A B C$ representing lattice planes with Miller indices (111) in Fig. 6. It will be noted that the lattice nodes are uniformly distributed in the lattice plane.

If the lattice (Fig. 6 ) is expressed as a primitive lattice, there is half an open circle [3( $\pi / 3)$ out of $2 \pi]$, thus half a lattice node within triangle $A B C$ which has an area of $S_{A B C}$.

2 Fig. F1 Open circles represent lattice nodes. The angle $2 \pi$ represents a complete circle of the node. The circle at the bottom right of (a) is expanded in (b) to illustrate how it is shared by four hexagonal unit cells while $\pi$ (c), $2 \pi / 3$ (d), and $\pi / 3$ (e) represent $1 / 2$, $1 / 3$, and $1 / 6$ of a circle, shared by two, three, and six hexagonal unit cells, respectively, (e) also indicates a circle shared by six triangles. The same principle can be expanded to a three-dimensional lattice with a sphere representing for a node.

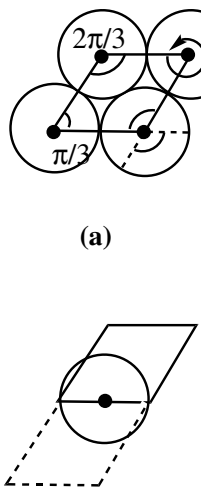

(c)

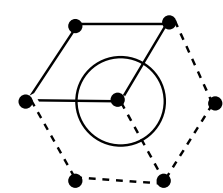

(d)

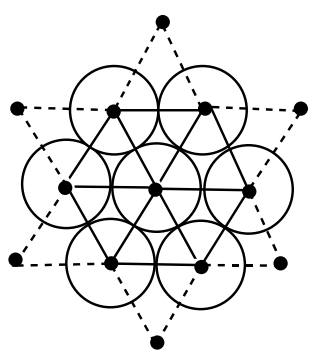

(e) 


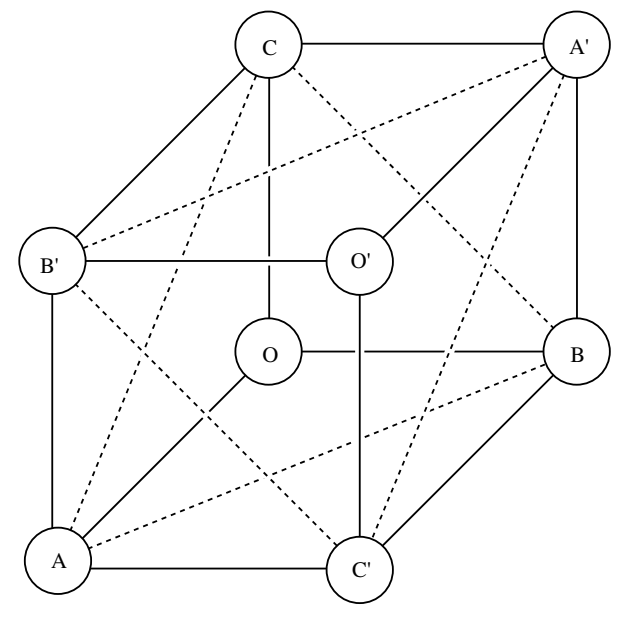

Fig. 7 A simple cubic unit cell with only one lattice node representing a conventional primitive lattice. A non-primitive lattice can be constructed by using a non-conventional body-centered rhombohedral cell with axes $\mathbf{O A}^{\prime}, \mathbf{O} \mathbf{B}^{\prime}$, and $\mathbf{O C}^{\prime}$

$$
\begin{aligned}
S_{A B C} & =\frac{1}{2}(\mathbf{B C} \times \mathbf{A B})=\frac{1}{2}|\mathbf{B C}|\left(\frac{\sqrt{3}}{2}|\mathbf{A B}|\right) \\
& =\frac{1}{2}\left(\frac{\sqrt{2}}{2}\left|\mathbf{O A}^{\prime}\right|\right)\left(\frac{\sqrt{3}}{2} \frac{\sqrt{2}}{2}\left|\mathbf{O A}^{\prime}\right|\right) \\
& =\frac{\sqrt{3}}{8}\left|\mathbf{O A}^{\prime}\right|^{2}
\end{aligned}
$$

When the same lattice is expressed as a face-centered cubic lattice, there are two open circles $[3(\pi / 3)+3 \pi$ out of $2 \pi]$ or two lattice nodes [the open circle at the vertex contributes $1 / 6$ to the triangle $A^{\prime} B^{\prime} C^{\prime}$ and the circle on the edge contributes 1/2.] within triangle $A^{\prime} B^{\prime} C^{\prime}$ which has an area of $S_{A^{\prime} B^{\prime} C^{\prime}}$

$$
\begin{aligned}
S_{A^{\prime} B^{\prime} C^{\prime}} & =\frac{1}{2}\left|\mathbf{A}^{\prime} \mathbf{B}^{\prime}\right|\left|\mathbf{C}^{\prime} \mathbf{C}\right| \\
& =\frac{1}{2}\left(\sqrt{2}\left|\mathbf{O} \mathbf{A}^{\prime}\right|\right)\left(\frac{\sqrt{3}}{2} \sqrt{2}\left|\mathbf{O A}^{\prime}\right|\right) \\
& =\frac{\sqrt{3}}{2}\left|\mathbf{O A}^{\prime}\right|^{2}
\end{aligned}
$$

It is apparent from these calculations that the lattice node density (lattice points per unit area) for the lattice plane ( $A B C$ or $A^{\prime} B^{\prime} C^{\prime}$ in Fig. 6) with Miller indices (111) is not changed even though the unit cell representation is changed from primitive to non-primitive. Thus, the lattice node density of a lattice plane in a non-primitive lattice can be obtained directly from its alternative primitive lattice representation. It is totally unnecessary to use the $P D$ number defined in [4] to recalculate the lattice node density for the lattice plane of a non-primitive lattice. To calculate the lattice node density for lattice planes with
Miller indices $(h k l)$ of the non-conventional body-centered rhombohedral lattice in Fig. 7, it is only necessary to calculate the lattice node density for the same family of lattice planes in the corresponding primitive cubic lattice.

Eqs. 17 and 18 represent relationships between the axes of a non-primitive rhombohedral lattice and the related primitive cubic lattice as shown in Fig. 7.

$\left(\begin{array}{l}\mathbf{a}^{\prime} \\ \mathbf{b}^{\prime} \\ \mathbf{c}^{\prime}\end{array}\right)=\left(\begin{array}{lll}0 & 1 & 1 \\ 1 & 0 & 1 \\ 1 & 1 & 0\end{array}\right)\left(\begin{array}{l}\mathbf{a} \\ \mathbf{b} \\ \mathbf{c}\end{array}\right)$

$\left(\begin{array}{l}\mathbf{a} \\ \mathbf{b} \\ \mathbf{c}\end{array}\right)=\left(\begin{array}{lll}0 & 1 & 1 \\ 1 & 0 & 1 \\ 1 & 1 & 0\end{array}\right)^{-1}\left(\begin{array}{l}\mathbf{a}^{\prime} \\ \mathbf{b}^{\prime} \\ \mathbf{c}^{\prime}\end{array}\right)=\frac{1}{2}\left(\begin{array}{ccc}-1 & 1 & 1 \\ 1 & -1 & 1 \\ 1 & 1 & -1\end{array}\right)\left(\begin{array}{l}\mathbf{a}^{\prime} \\ \mathbf{b}^{\prime} \\ \mathbf{c}^{\prime}\end{array}\right)$

where $\mathbf{a}, \mathbf{b}$, and $\mathbf{c}$ are $\mathbf{O A}, \mathbf{O B}$, and $\mathbf{O C}$ while $\mathbf{a}^{\prime}, \mathbf{b}^{\prime}$, and $\mathbf{c}^{\prime}$ are $\mathbf{O} \mathbf{A}^{\prime}, \mathbf{O} \mathbf{B}^{\prime}$, and $\mathbf{O} \mathbf{C}^{\prime}$. The volume $V_{a b c}$ of the unit cell and interplanar spacing between planes with Miller indices ( $h k l)$ in a primitive lattice can be obtained using either Eqs. 17 or 18. The density of lattice nodes in a lattice plane can be determined from this volume and the interplanar spacing. The volume of this cubic unit cell is given by Eq. 19 [39].

$V_{a b c}=\mathbf{a} \cdot(\mathbf{b} \times \mathbf{c})=|\mathbf{a}||\mathbf{b}||\mathbf{c}|=\frac{\left|\mathbf{a}^{\prime}\right|}{\sqrt{2}} \frac{\left|\mathbf{b}^{\prime}\right|}{\sqrt{2}} \frac{\left|\mathbf{c}^{\prime}\right|}{\sqrt{2}}=\frac{\left|\mathbf{a}^{\prime}\right|^{3}}{2 \sqrt{2}}$

The relationship between the volumes [39] for the primitive cubic lattice $V_{a b c}$ and the related non-primitive lattice $V_{a^{\prime} b^{\prime} c^{\prime}}$ can be obtained from Eq. 3.

$$
\begin{aligned}
V_{a^{\prime} b^{\prime} c^{\prime}} & =\mathbf{a}^{\prime} \cdot\left(\mathbf{b}^{\prime} \times \mathbf{c}^{\prime}\right)=\left|\left(\begin{array}{ccc}
0 & |\mathbf{b}| & |\mathbf{c}| \\
|\mathbf{a}| & 0 & |\mathbf{c}| \\
|\mathbf{a}| & |\mathbf{b}| & 0
\end{array}\right)\right| \\
& \left.=\left|\left(\begin{array}{lll}
0 & 1 & 1 \\
1 & 0 & 1 \\
1 & 1 & 0
\end{array}\right)\right||\mathbf{a}||\mathbf{b}||\mathbf{c}|=\mid \begin{array}{lll}
0 & 1 & 1 \\
1 & 0 & 1 \\
1 & 1 & 0
\end{array}\right)\left.|| \mathbf{a}\right|^{3} \\
& =2|\mathbf{a}|^{3}=2[\mathbf{a} \cdot(\mathbf{b} \times \mathbf{c})]=2 V_{a b c}
\end{aligned}
$$

or

$$
\begin{aligned}
V_{a^{\prime} b^{\prime} c^{\prime}}= & \mathbf{a}^{\prime} \cdot\left(\mathbf{b}^{\prime} \times \mathbf{c}^{\prime}\right)=\mathbf{c}^{\prime} \cdot\left(\mathbf{a}^{\prime} \times \mathbf{b}^{\prime}\right) \\
& =\left|\mathbf{c}^{\prime}\right|\left(\left|\mathbf{a}^{\prime}\right|\left|\mathbf{b}^{\prime}\right| \sin \gamma^{\prime}\right) \cos \theta^{\prime} \\
& =\left|\mathbf{c}^{\prime}\right|\left(\left|\mathbf{a}^{\prime}\right|\left|\mathbf{b}^{\prime}\right| \sin \gamma^{\prime}\right) \\
& \sqrt{\frac{\left|1-\cos ^{2} \alpha^{\prime}-\cos ^{2} \beta^{\prime}-\cos ^{2} \gamma^{\prime}+2 \cos \alpha^{\prime} \cos \beta^{\prime} \cos \gamma^{\prime}\right|}{\sin ^{2} \gamma^{\prime}}} \\
= & \frac{1}{\sqrt{2}}\left|\mathbf{a}^{\prime}\right|\left|\mathbf{b}^{\prime}\right|\left|\mathbf{c}^{\prime}\right|=\frac{1}{\sqrt{2}}\left|\mathbf{a}^{\prime}\right|^{3}=2|\mathbf{a}|^{3}=2 V_{a b c}
\end{aligned}
$$

$\alpha^{\prime}, \beta^{\prime}, \gamma^{\prime}$ are the angles ( $\pi / 3$ in this case) between the axes $\mathbf{b}^{\prime}$ and $\mathbf{c}^{\prime}, \mathbf{c}^{\prime}$ and $\mathbf{a}^{\prime}$, and $\mathbf{a}^{\prime}$ and $\mathbf{b}^{\prime}$, respectively. $\theta^{\prime}$ is the angle between $\mathbf{c}^{\prime}$ and its reciprocal vector $\mathbf{c}^{\prime *}$ shown in 
Fig. 1 [39]. When the vectors are expressed in a Cartesian coordinate system, Eq. 21 can be obtained more generally from Eq. 17 or 18 [13].

$$
\begin{aligned}
& V_{a b c}^{2}=[\mathbf{a} \cdot(\mathbf{b} \times \mathbf{c})]^{2}=\left|\left(\begin{array}{lll}
a_{x} & b_{x} & c_{x} \\
a_{y} & b_{y} & c_{y} \\
a_{z} & b_{z} & c_{z}
\end{array}\right)\left(\begin{array}{lll}
a_{x} & a_{y} & a_{z} \\
b_{x} & b_{y} & b_{z} \\
c_{x} & c_{y} & c_{z}
\end{array}\right)\right| \\
& =\mid\left(\begin{array}{llll}
a_{x}{ }^{\prime} & b_{x}{ }^{\prime} & c_{x}{ }^{\prime} \\
a_{y}{ }^{\prime} & b_{y}^{\prime} & c_{y}{ }^{\prime} \\
a_{z}{ }^{\prime} & b_{z}^{\prime} & c_{z}{ }^{\prime}
\end{array}\right)\left(\begin{array}{ccc}
-1 / 2 & 1 / 2 & 1 / 2 \\
1 / 2 & -1 / 2 & 1 / 2 \\
1 / 2 & 1 / 2 & -1 / 2
\end{array}\right) \\
& \left(\begin{array}{ccc}
-1 / 2 & 1 / 2 & 1 / 2 \\
1 / 2 & -1 / 2 & 1 / 2 \\
1 / 2 & 1 / 2 & -1 / 2
\end{array}\right)\left(\begin{array}{lll}
a_{x}{ }^{\prime} & a_{y}{ }^{\prime} & a_{z}{ }^{\prime} \\
b_{x}{ }^{\prime} & b_{y}^{\prime} & b_{z}{ }^{\prime} \\
c_{x}{ }^{\prime} & c_{y}{ }^{\prime} & c_{z}{ }^{\prime}
\end{array}\right) \mid \\
& =\frac{1}{4}\left|\left(\begin{array}{ccc}
a_{x}^{\prime} & b_{x}^{\prime} & c_{x}{ }^{\prime} \\
a_{y}^{\prime} & b_{y}^{\prime} & c_{y}{ }^{\prime} \\
a_{z}^{\prime} & b_{z}^{\prime} & c_{z}^{\prime}
\end{array}\right)\left(\begin{array}{lll}
a_{x}^{\prime} & a_{y}^{\prime} & a_{z}^{\prime} \\
b_{x}^{\prime} & b_{y}^{\prime} & b_{z}^{\prime} \\
c_{x}^{\prime} & c_{y}^{\prime} & c_{z}^{\prime}
\end{array}\right)\right| \\
& =\frac{1}{4}\left[\mathbf{a}^{\prime} \cdot\left(\mathbf{b}^{\prime} \times \mathbf{c}^{\prime}\right)\right]^{2}=\frac{1}{4} V_{a^{\prime} b^{\prime} c^{\prime}}^{2} \\
& V_{a b c}=\mathbf{a} \cdot(\mathbf{b} \times \mathbf{c})=\left|\left(\begin{array}{lll}
a_{x} & a_{y} & a_{z} \\
b_{x} & b_{y} & b_{z} \\
c_{x} & c_{y} & c_{z}
\end{array}\right)\right| \\
& =\left|\left(\begin{array}{ccc}
-1 / 2 & 1 / 2 & 1 / 2 \\
1 / 2 & -1 / 2 & 1 / 2 \\
1 / 2 & 1 / 2 & -1 / 2
\end{array}\right)\left(\begin{array}{lll}
a_{x}^{\prime} & a_{y}^{\prime} & a_{z}^{\prime} \\
b_{x}^{\prime} & b_{y}^{\prime} & b_{z}^{\prime} \\
c_{x}^{\prime} & c_{y}^{\prime} & c_{z}^{\prime}
\end{array}\right)\right| \\
& =\frac{1}{2}\left|\left(\begin{array}{ccc}
a_{x}{ }^{\prime} & a_{y}{ }^{\prime} & a_{z}{ }^{\prime} \\
b_{x}{ }^{\prime} & b_{y}{ }^{\prime} & b_{z}{ }^{\prime} \\
c_{x}^{\prime} & c_{y}^{\prime} & c_{z}{ }^{\prime}
\end{array}\right)\right|=\frac{1}{2} \mathbf{a}^{\prime} \cdot\left(\mathbf{b}^{\prime} \times \mathbf{c}^{\prime}\right)=\frac{1}{2} V_{a^{\prime} b^{\prime} c^{\prime}}
\end{aligned}
$$

To calculate the interplanar spacing between planes with Miller indices ( $h k l$ in the primitive cubic lattice, we can first convert the Miller indices from $\left(h^{\prime} k^{\prime} l^{\prime}\right)$ in the non-primitive rhombohedral lattice to ( $h k l)$ using Eqs. 17 or 18. The Miller indices can be transformed (Eq. 24) in the same way as the axes [39].

$\left(\begin{array}{l}h \\ k \\ l\end{array}\right)=\frac{1}{2}\left(\begin{array}{ccc}-1 & 1 & 1 \\ 1 & -1 & 1 \\ 1 & 1 & -1\end{array}\right)\left(\begin{array}{l}h^{\prime} \\ k^{\prime} \\ l^{\prime}\end{array}\right)$

For the plane with Miller indices (011) in the non-primitive rhombohedral lattice, its Miller indices can be converted to (100) for the primitive cubic lattice by Eq. 25 or 26.

$$
\begin{aligned}
& \left(\begin{array}{l}
h \\
k \\
l
\end{array}\right)=\frac{1}{2}\left(\begin{array}{ccc}
-1 & 1 & 1 \\
1 & -1 & 1 \\
1 & 1 & -1
\end{array}\right)\left(\begin{array}{l}
0 \\
1 \\
1
\end{array}\right)=\left(\begin{array}{l}
1 \\
0 \\
0
\end{array}\right) \\
& \left(\begin{array}{l}
h^{\prime} \\
k^{\prime} \\
l^{\prime}
\end{array}\right)=\left(\begin{array}{lll}
0 & 1 & 1 \\
1 & 0 & 1 \\
1 & 1 & 0
\end{array}\right)\left(\begin{array}{l}
1 \\
0 \\
0
\end{array}\right)=\left(\begin{array}{l}
0 \\
1 \\
1
\end{array}\right)
\end{aligned}
$$

The interplanar spacing $d_{100}$ for the planes with Miller indices (100) in the primitive cubic lattice is

$$
\begin{aligned}
d_{100} & =1 / \sqrt{\left(\frac{h}{|\mathbf{a}|}\right)^{2}+\left(\frac{k}{|\mathbf{a}|}\right)^{2}+\left(\frac{l}{|\mathbf{a}|}\right)^{2}} \\
& =1 / \sqrt{\left(\frac{1}{|\mathbf{a}|}\right)^{2}+0+0}=|\mathbf{a}|=|\mathbf{b}|=|\mathbf{c}|
\end{aligned}
$$

Since there is only one lattice node within square $A B^{\prime} O^{\prime}$ $C^{\prime}$ in Fig. 7, the lattice node density for this plane can be calculated from the lattice nodes per unit area within this square.

$$
\begin{aligned}
\rho\left(S_{100}, C P\right) & =\frac{1}{S_{100}}=\frac{1}{V_{a b c} / d_{100}} \\
& =\frac{|\mathbf{a}|}{|\mathbf{a}||\mathbf{b}||\mathbf{c}|}=\frac{1}{|\mathbf{b}||\mathbf{c}|}=\frac{1}{|\mathbf{a}|^{2}}
\end{aligned}
$$

By converting the non-primitive lattice to a primitive lattice the tedious calculations for PD numbers in Fan's method can be avoided since there is only one lattice point in a primitive unit cell. The conversion can be achieved easily using Eqs. 17 and 18. The $d_{h k l}$ involved in this method can be readily obtained from the conventional method, [39] while the method using Table 3 in [5] is considerably more lengthy, complicated, and error prone than the method presented here.

\subsection{The problems with Fan's method shown by examples}

Other than the unjustified claim that the densities of lattice nodes for a set of parallel lattice planes could be different, this section shows other problems with Fan's method. If we use the method of $P D$ numbers [4], the interplanar spacing $d_{h k l}^{\prime}$ for planes with Miller indices $\left(h^{\prime} k^{\prime} l^{\prime}\right)$ in a nonprimitive lattice needs to be calculated along with the calculation of $V_{a^{\prime} b^{\prime} c^{\prime}}$, which is similar to our method using a primitive lattice. From the reciprocal vector method [39], we have Eq. 29 for the rhombohedral lattice.

$$
\begin{aligned}
d_{h k l} & =\sqrt{\frac{|\mathbf{a}|^{2} \sin ^{2} \alpha \sin ^{2} \alpha^{*}}{\left(h^{2}+k^{2}+I^{2}\right)+2(h k+k l+I h) \cos \alpha^{*}}} \\
& =\sqrt{\frac{|\mathbf{a}|^{2} \sin ^{2} \alpha \frac{\left(1-3 \cos ^{2} \alpha+2 \cos ^{3} \alpha\right)}{\sin ^{4} \alpha}}{\left(h^{2}+k^{2}+l^{2}\right)+2(h k+k l+I h) \frac{\left(\cos ^{2} \alpha-\cos \alpha\right)}{\sin ^{2} \alpha}}} \\
& =\sqrt{\frac{|\mathbf{a}|^{2}\left(1-3 \cos ^{2} \alpha+2 \cos ^{3} \alpha\right)}{\left(h^{2}+k^{2}+l^{2}\right) \sin ^{2} \alpha-2(h k+k l+l h)\left(\cos \alpha-\cos ^{2} \alpha\right)}}
\end{aligned}
$$

Let us consider the example of Miller indices (110) in the non-primitive lattice shown in Fig. 7. 
$d_{110}^{\prime}=\sqrt{\frac{\left|\mathbf{a}^{\prime}\right|^{2}\left(1-\frac{3}{4}+\frac{2}{8}\right)}{(1+1+0) \frac{3}{4}-2(0+0+1)\left(\frac{1}{2}-\frac{1}{4}\right)}}=\frac{\sqrt{2}}{2}\left|\mathbf{a}^{\prime}\right|$

The additional work necessary with Fan's method involves calculating $P D$ numbers as follows. Eq. 31 is the equation for planes with Miller indices $\left(h^{\prime} k^{\prime} l^{\prime}\right)$. For a primitive lattice, $r$ is an integer $n$, and Eq. 31 reduces to Eq. 4. For a non-primitive lattice, $r$ is a rational number and for atomic plane $r$ can be a real number but this last possibility is ignored in [4]. All points with the same value of $r$ are on the same plane.

$h^{\prime} x^{\prime}+k^{\prime} y^{\prime}+l^{\prime} z^{\prime}=r$

$x^{\prime}, y^{\prime}$, and $z^{\prime}$ in Eq. 31 are the coordinates of a lattice node in the non-primitive lattice or of an atom in a crystal. Equation 31 is quite general and applies to primitive (where $r$ is an integer), non-primitive (where $r=m / q$ with $m$ and $q$ integers) with $q$ be greater than 1 , and crystals ( $r$ can be any real number not just limited to $m / q$ ).

For the non-primitive rhombohedral lattice shown in Fig. 7, there are two lattice points $\mathrm{O}$ and $\mathrm{O}^{\prime}$ with coordinates $(0,0,0)$ and $(1 / 2,1 / 2,1 / 2)$ in the unit cell. For the planes with Miller indices (110), the value of $r$ can be calculated using Eq. 31

$1 \times 0+1 \times 0+0 \times \frac{1}{2}=0$

$1 \times \frac{1}{2}+1 \times \frac{1}{2}+0 \times \frac{1}{2}=1$

Those points in the unit cell with integral values of $r$ can be translated onto the same plane. For example, $\mathrm{O}$ in Fig. 7 can be translated to $A^{\prime}$ or $B^{\prime}$ which along with $O^{\prime}$ and $C$ are contained within the (110) plane of $O^{\prime} A^{\prime} C B^{\prime}$ for the non-primitive rhombohedral lattice. Since both the lattice points $O$ and $O^{\prime}$ are within the unit cell, they should be included when the $P D$ number is calculated.

$$
\begin{aligned}
\rho\left(S_{110}^{\prime}\right) & =\frac{2}{S_{110}^{\prime}}=\frac{2}{V_{a^{\prime} b^{\prime} c^{\prime}} / d_{110}^{\prime}} \\
& =\frac{2 d_{110}^{\prime}}{V_{a^{\prime} b^{\prime} c^{\prime}}}=(P D) \frac{d_{110}^{\prime}}{V_{a^{\prime} b^{\prime} c^{\prime}}} \\
& =\frac{2 \frac{1}{\sqrt{2}}\left|\mathbf{a}^{\prime}\right|}{\frac{1}{\sqrt{2}}\left|\mathbf{a}^{\prime}\right|^{3}}=\frac{1}{\left|\frac{1}{\sqrt{2}} \mathbf{a}^{\prime}\right|^{2}}=\frac{1}{|\mathbf{a}|^{2}}
\end{aligned}
$$

Within the I-centered non-primitive cell, there are two lattice points in the plane with Miller indices (110) which are represented by the 2 in the numerator above $S_{110}^{\prime}$ in Eq. 34 which is defined as the $P D$ number. When there are several lattice nodes in a unit cell, the calculations for $P D$ number can be tedious and error prone. By contrast, in the simpler method using the primitive lattice there is no need to calculate the $P D$ number.

It can be seen from Eqs. 28 and 34 that the density of nodes in the plane $\mathrm{O}^{\prime} \mathrm{A}^{\prime} \mathrm{CB}^{\prime}$ in Fig. 7 does not change when the lattice is transformed from a primitive to a non-primitive representation. The result from Fan's method seems correct but cannot be recommended not only because of the tedious calculations that are necessary to obtain the $P D$ number for Eq. 34 but also because it can lead to significant errors (see below).

With similar calculations like Eqs. 25 and 26, it can be seen that the Miller indices (111) remain unchanged when the unit cell is transformed between the primitive and non-primitive representation shown in Fig. 7. The Miller indices (111) in an rhombohedral unit cell transform to $(1 / 2,1 / 2,1 / 2)$ in a cubic unit cell. But of course $(1 / 2,1 / 2$, $1 / 2$ ) should be converted to (111) using the conventional definition of Miller indices. The conversion is indicated by an arrow in Eq. 35 since the process has not been imbedded in the mathematical expressions of Eqs. 25 and 26.

$\left(\begin{array}{l}h \\ k \\ l\end{array}\right)=\frac{1}{2}\left(\begin{array}{ccc}-1 & 1 & 1 \\ 1 & -1 & 1 \\ 1 & 1 & -1\end{array}\right)\left(\begin{array}{l}1 \\ 1 \\ 1\end{array}\right) \Rightarrow\left(\begin{array}{l}1 \\ 1 \\ 1\end{array}\right)$

Although there are only two lattice points $\mathrm{O}(0,0,0)$ and $\mathrm{O}^{\prime}(1 / 2,1 / 2,1 / 2)$ in the unit cell for the non-primitive rhombohedral lattice, there are more than two planes with Miller indices (111) passing through the unit cell, a fact which causes a problem in obtaining the value of the $P D$ number. The two planes passing through points $\mathrm{O}$ and $\mathrm{O}^{\prime}$ in Fig. 7, have values of $r$ of 0 and 3/2, respectively.

$h^{\prime} x^{\prime}+k^{\prime} y^{\prime}+l^{\prime} z^{\prime}=1 \times 0+1 \times 0+1 \times 0=0$

$h^{\prime} x^{\prime}+k^{\prime} y^{\prime}+l^{\prime} z^{\prime}=1 \times \frac{1}{2}+1 \times \frac{1}{2}+1 \times \frac{1}{2}=\frac{3}{2}$

Equation 37 for $\mathrm{O}^{\prime}$ can also be evaluated from Eqs. 9 in terms of $\mathbf{a}, \mathbf{b}$, and $\mathbf{c}$ via Eqs. 38 and 39 [39]. The position of $\mathrm{O}^{\prime}$ can be obtained from Eq. 17.

$$
\begin{aligned}
x^{\prime} \mathbf{a}^{\prime}+y^{\prime} \mathbf{b}^{\prime}+z^{\prime} \mathbf{c}^{\prime} & =\frac{1}{2}(\mathbf{b}+\mathbf{c}) \times \frac{1}{2}(\mathbf{a}+\mathbf{c})+\frac{1}{2}(\mathbf{a}+\mathbf{b}) \\
& =\mathbf{a}+\mathbf{b}+\mathbf{c}
\end{aligned}
$$

The unit vector perpendicular to planes with Miller indices (111) is 


$$
\begin{aligned}
h^{\prime} \mathbf{a}^{\prime *}+k^{\prime} \mathbf{b}^{\prime *}+l^{\prime} \mathbf{c}^{\prime *}= & \frac{\mathbf{b}^{\prime} \times \mathbf{c}^{\prime}}{2 V_{a b c}}+\frac{\mathbf{c}^{\prime} \times \mathbf{a}^{\prime}}{2 V_{a b c}}+\frac{\mathbf{a}^{\prime} \times \mathbf{b}^{\prime}}{2 V_{a b c}} \\
= & \frac{(\mathbf{a}+\mathbf{c}) \times(\mathbf{a}+\mathbf{b})}{2 V_{a b c}}+\frac{(\mathbf{a}+\mathbf{b}) \times(\mathbf{b}+\mathbf{c})}{2 V_{a b c}} \\
& +\frac{(\mathbf{b}+\mathbf{c}) \times(\mathbf{a}+\mathbf{c})}{2 V_{a b c}} \\
= & \frac{\mathbf{a} \times \mathbf{b}+\mathbf{c} \times \mathbf{a}+\mathbf{c} \times \mathbf{b}}{2 V_{a b c}}+\frac{\mathbf{a}^{*}-\mathbf{b}^{*}+\mathbf{c}^{*}}{2} \\
& +\frac{\mathbf{a}^{*}+\mathbf{b}^{*}-\mathbf{c}^{*}}{2} \\
= & \frac{\mathbf{a}^{*}+\mathbf{b}^{*}+\mathbf{c}^{*}}{2}
\end{aligned}
$$

The lattice plane for $\mathrm{O}^{\prime}$ characterized by Miller indices $\left(h^{\prime} k^{\prime} l^{\prime}\right)$ is

$$
\begin{aligned}
h^{\prime} x^{\prime}+k^{\prime} y^{\prime}+l^{\prime} z^{\prime} & =\left(x^{\prime} \mathbf{a}^{\prime}+y^{\prime} \mathbf{b}^{\prime}+z^{\prime} \mathbf{c}^{\prime}\right) \cdot\left(h^{\prime} \mathbf{a}^{* *}+k^{\prime} \mathbf{b}^{\prime *}+l^{\prime} \mathbf{c}^{\prime *}\right) \\
& =(\mathbf{a}+\mathbf{b}+\mathbf{c}) \cdot \frac{1}{2}\left(\mathbf{a}^{*}+\mathbf{b}^{*}+\mathbf{c}^{*}\right)=\frac{3}{2}
\end{aligned}
$$

The results of Eqs. 37 and 40 are the same. There are also planes with $r=1 / 2$ for plane $A B C$ and $r=1$ for $A^{\prime} B^{\prime} C^{\prime}$ in Fig. 7. For $C^{\prime}(0,0,1)$ with plane $A^{\prime} B^{\prime} C^{\prime}$

$h^{\prime} x^{\prime}+k^{\prime} y^{\prime}+l^{\prime} z^{\prime}=1 \times 0+1 \times 0+1 \times 1=1$

For $C(1 / 2,1 / 2,-1 / 2)$ with plane $A B C$

$h^{\prime} x^{\prime}+k^{\prime} y^{\prime}+l^{\prime} z^{\prime}=1 \times \frac{1}{2}+1 \times \frac{1}{2}+1 \times\left(-\frac{1}{2}\right)=\frac{1}{2}$

or

$x^{\prime} \mathbf{a}^{\prime}+y^{\prime} \mathbf{b}^{\prime}+z^{\prime} \mathbf{c}^{\prime}=\frac{1}{2}(\mathbf{b}+\mathbf{c}) \times \frac{1}{2}(\mathbf{a}+\mathbf{c})-\frac{1}{2}(\mathbf{a}+\mathbf{b})=\mathbf{c}$

$h^{\prime} x^{\prime}+k^{\prime} y^{\prime}+l^{\prime} z^{\prime}=\mathbf{c} \cdot \frac{1}{2}\left(\mathbf{a}^{*}+\mathbf{b}^{*}+\mathbf{c}^{*}\right)=\frac{1}{2}$

In Fan's method, the $P D$ number is calculated using the number of points in a unit cell and as a result parallel lattice planes could have different point densities. These requirements have made the method problematic. With Fan's method, these latter two planes with $r=1$ and 1/2 can be missing. The node on a plane cannot be accounted for twice even though there are more than one plane with integral values of $r$. There are only 2 lattice nodes in the unit cell. $\mathrm{O}^{\prime}$ is contained within the plane with $r=3 / 2$ and it cannot be assigned to the plane with $r=1 / 2$. And $O$ belongs to the plane with $r=0$, and it cannot be assigned to the plane with $r=1$. Thus, the $P D$ number is 1 for both planes with $r=0$ and $3 / 2$ and it is 0 for planes with $r=1 / 2$ and 1 in Fan's method which makes it difficult to use the method satisfactorily.
The PD number calculated with Fan's method [4] is 1 for each of the planes with $r=0$ and $r=3 / 2$. Converting from the primitive cubic lattice to the non-primitive rhombohedral lattice in Fig. 7, the unit cell volume is increased by a factor of 2, while the interplanar spacing has not changed $d_{111}$ (cubic) $=d_{111}$ (rhombohedral). Thus, using a similar formula to that of Eq. 34 from Fan's method, the calculated lattice node density can be wrong because the calculated value is smaller by a factor of 2 than the actual value for planes with $r=0$ and 3/2 since for each of these planes the $P D$ number is calculated as 1 instead of 2 . The planes with $r=1 / 2$ and 1 are missing since the $P D$ number is 0 for each of these planes or to put it another way the node density for these planes is 0 . Thus, two different node densities are obtained for the (111) planes for the non-primitive lattice in Fan's method. What is wrong will be discussed in more detail in Sect. 3.4, but here we note that since the volume is increased by a factor of 2 , the base area perpendicular to $d_{111}$ should also be increased by a factor of 2 to accommodate one more lattice point in the base area and thus the node density for (111) planes should be the same for the primitive and the centered lattices. However, the problem inherent in Fan's method can readily be overcome by converting the non-primitive lattice to the primitive lattice.

\subsection{Node density for crystal planes}

Fan's article [4] states that in a non-primitive lattice the node density can be different for lattice planes $(h k l)$ with different values of $m$ in Eqs. 14 or 31 where $r$ could only be $m / q$ and both $m$ and $q$ are integers. As pointed out above, every lattice plane from a family will have the same lattice node density whether it is described within a primitive or non-primitive lattice.

Atoms located exactly at the lattice nodes of a facecentered cubic cell count as 4 atoms within the conventional unit cell as the majority are shared with adjacent unit cells. When atoms within a crystal are concerned, then Eq. 31 can be applied with $r=n+\delta$ where $n$ is an integer and $\delta$ can be any real number representing the perpendicular distance of the atom from a lattice plane with Miller indices $(h k l)$. A problem with Fan's article [4] is that it is stated that $\delta$ takes the value of $m / q$, but that is not necessarily true. When the atomic coordinates are inserted into Eq. 31, $n+\delta$ can be evaluated for planes with specific Miller indices. All the atoms within a crystal unit cell that have the same value of $n+\delta$ with the specified Miller indices are located on the same crystal plane. It can be considered in an equivalent manner to $r=0$ when $n+\delta$ calculated from the atoms within the unit cell has an integral value. The atoms with the same values of $n+\delta$ within a crystal unit cell, even if they, at first sight, seem to 
be on different planes, can be translated to become nearest neighbors in the same plane thus all of them together form a two-dimension unit cell. The whole crystal plane can be constructed from this two-dimensional unit cell by periodicity. It is apparent that the point density for the plane in crystal can be calculated using atomic positions in this two-dimensional unit cell.

\subsection{Relative node density for lattice planes with different Miller indices}

The lattice node density for a lattice plane in a lattice cannot be changed even if the lattice representation is changed from a primitive cell to a non-primitive cell. The lattice does not change whatever the representation of the unit cell. Each of the different unit cells fully characterize the same lattice. Thus, the lattice node density for planes with any Miller indices can be determined if the density of another specific plane with different Miller indices is known.

We have shown that the lattice node density for nonprimitive lattice planes can be easily calculated by transforming the non-primitive lattice into its equivalent primitive lattice. The calculations apply to conventional as well as non-conventional lattices. The advantage of this method is that the relative lattice node density can be easily calculated for lattice planes with different Miller indices and the method developed here for primitive lattices is applicable to non-primitive lattices.

A primitive unit cell in a lattice only contains a single lattice node, so a unit cell which contains two or more lattice nodes is not primitive. Thus, all different primitive unit cells in a lattice (Fig. 8) have the same volume, and this volume represents a single lattice node and all the planes with different Miller indices can be represented by a plane passing

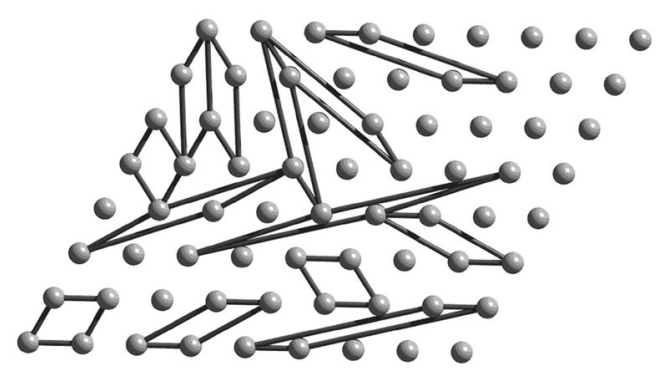

Fig. 8 A two-dimensional lattice. Based on the lattice lines and the interlinear spacing between parallel lines, there is an infinite number of choices for a primitive unit cell. Similarly, all the primitive unit cells in a three-dimensional lattice contain only one lattice node and thus all the cells have the same volume. This is because the lattice nodes are uniformly distributed and the bulk density of the lattice is fixed. The bulk density of the lattice can be calculated from any one of the primitive unit cells through the origin $(0,0,0)$. Therefore, the node density for a lattice plane $\rho\left(S_{h k l}\right)$ with Miller indices $(h k l)$ is given by

$\rho\left(S_{h k l}\right)=\frac{1}{S_{h k l}}=\frac{1}{V_{h k l} / d_{h k l}}=\frac{d_{h k l}}{V_{h k l}}$

If each lattice line represents a lattice plane perpendicular to the paper, Fig. 8 represents a three dimensional lattice. Each of the primitive unit cells shown in Fig. 8 is then characterized by a family of parallel planes with specific Miller indices and its interplanar spacing, if the lattice is three-dimensional. No matter how the primitive unit cell is chosen for a specific lattice, the volume of the primitive unit cell $V_{h k l}$ in Eq. 45 is always the same. Thus, the node density for a lattice plane $\rho\left(S_{h k l}\right)$ can be determined from Eq. 45 when the interplanar spacing $d_{h k l}$ for planes with Miller indices $(h k l)$ is known.

The principle implied in Eq. 45 is simple. The lattice node density for a bulk lattice is the number of lattice nodes per unit volume. The primitive unit cell contains only one lattice node. The different choice of primitive unit cell is reflected by Eq. 46 with a base area $S_{h k l}$ and a height $d_{h k l}$, i.e., the volume can be calculated from Eq. 46 with a base area $S_{h k l}$ and a height $d_{h k l}$ by different choice of primitive unit cell. When $d_{h k l}$ and the bulk density is known, the base area $S_{h k l}$ can be determined.

$V_{h k l}=S_{h k l} d_{h k l}=1$

When the interplanar spacing $d_{h k l}$ is reduced by a factor of $N$ for planes from Miller indices $\left(h_{1} k_{1} l_{1}\right)$ to $\left(h_{2} k_{2} l_{2}\right)$ in a primitive unit cell, the base area will be increased by a factor of $N$ since the volume of the primitive unit cell is fixed for a specific lattice. Since there is only one lattice node in the primitive unit cell, the lattice node density for the new plane $\rho(S)$ will be reduced by a factor of $N$. This value of lattice node density for a plane is consistent with the fact that the choice of unit cell cannot affect the lattice node density of the bulk lattice.

\subsubsection{The simple cubic lattice}

The node density for a lattice plane $\rho\left(S_{100}\right)$ with Miller indices (100) in Fig. 7 for the conventional primitive lattice of simple cubic $C P$ is

$\rho\left(S_{100}, C P\right)=\frac{1}{S_{O B A^{\prime} C}}=\frac{|\mathbf{O A}|}{S_{O B A^{\prime} C}|\mathbf{O A}|}=\frac{d_{100}(c P)}{V_{100}(C P)}$

$S_{O B A^{\prime} C}\left[=S_{100}(C P)\right]$ is the base area in the plane described by Miller indices (100) and $|\mathbf{O A}|\left[=d_{100}(C P)\right]$ is the height for the primitive unit cell related with the base plane (100). $V_{100}(C P)$ is the volume of this specific primitive unit 
cell. The same volume can be calculated for other primitive unit cells (Fig. 7) such as $V_{111}(C P)$ that with base area $S(\mathbf{A B} \times \mathbf{A C}, C P)$ or $S_{111}(C P)$ and height $d_{111}(C P)$ associated with Miller indices (111) [39].

$V_{100}(C P)=V_{111}(C P)=S_{111}(c P) d_{111}(C P)=(\mathbf{A B} \times \mathbf{A C}) \cdot \mathbf{O A}$

The relative lattice node density for two planes with different Miller indices can be determined by considering their relative interplanar spacing for the two families of planes as shown by Eq. 49, i.e., the lattice node density of the plane with Miller indices (111) can be obtained from the plane with Miller indices (100).

$$
\begin{aligned}
\frac{\rho\left(S_{111}, c P\right)}{\rho\left(S_{100}, c P\right)} & =\frac{1 / S_{111}(c P)}{1 / S_{100}(c P)}=\frac{S_{100}(c P)}{S_{111}(c P)} \\
& =\frac{d_{111}(c P) / V_{111}}{d_{100}(c P) / V_{100}}=\frac{d_{111}(c P)}{d_{100}(c P)} \\
& =\frac{|\mathbf{a}| / \sqrt{h^{\prime 2}+k^{\prime 2}+l^{\prime 2}}}{|\mathbf{a}| / \sqrt{h^{2}+k^{2}+l^{2}}} \\
& =\frac{|\mathbf{O B}| / \sqrt{h^{\prime 2}+k^{\prime 2}+l^{\prime 2}}}{|\mathbf{O B}| / h} \\
& =\frac{\frac{\sqrt{3}}{3}|\mathbf{O B}|}{|\mathbf{O B}|}=\frac{\sqrt{3}}{3}=N
\end{aligned}
$$

The bulk node density for a lattice is independent of the primitive unit cell selected. Since the height of the primitive unit cell $d_{111}(C P)$ is reduced to $\frac{\sqrt{3}}{3} d_{100}(C P)$ [= $\left.d_{111}=\frac{1}{3}\left|\mathbf{O O}^{\prime}\right|=\frac{1}{3}\left[\sqrt{3} d_{100}(C P)\right]\right][56]$ then the base area of the primitive unit cell $S_{111}(C P)$ must be increased to $\frac{3}{\sqrt{3}} S_{100}(C P)$ to keep the volume of the unit cell unchanged. Thus, the node density for the (111) plane $\rho\left(S_{111}, C P\right)$ is reduced to $N \rho\left(S_{100}, C P\right)$ or $\frac{\sqrt{3}}{3} \rho_{100}\left(S_{100}, C P\right)$. The result is correct since there is only one node $[4(\pi / 2)=2 \pi]$ within the square OBA' $C$ with base area $S_{100}(C P)=S_{O B A^{\prime} C}=|\mathbf{O B}|^{2}$; and there is $1 / 2$ a lattice node within triangle $A B C$ with base area $S_{111}(C P)=S_{A B C}$. The result is shown by Eq. 50 which acts as a check for Eq. 49.

$$
\begin{aligned}
\frac{\rho\left(S_{111}, C P\right)}{\rho\left(S_{100}, C P\right)} & =\frac{\frac{1 / 2}{\frac{1}{2}(|\mathbf{A B}|)\left(\frac{\sqrt{3}}{2}|\mathbf{A B}|\right)}}{1 /|\mathbf{O B}|^{2}} \\
& =\frac{\frac{1 / 2}{\frac{1}{2}(\sqrt{2}|\mathbf{O B}|)\left(\frac{\sqrt{3}}{2} \sqrt{2}|\mathbf{O B}|\right)}}{1 /|\mathbf{O B}|^{2}} \\
& =\frac{\frac{1 / 2}{\frac{1}{2} \sqrt{3}|\mathbf{O B}|^{2}}}{1 /|\mathbf{O B}|^{2}}=\frac{\sqrt{3}}{3}=N
\end{aligned}
$$

Although this method to calculate the relative node density for lattice planes is based on a primitive unit cell, it also applies to a non-primitive unit cell since the cell is fixed and the number of nodes in the cell is also fixed. The method applies not only to conventional primitive and non-primitive lattices, it can also be extended to nonconventional primitive and non-primitive lattices.

The above discussion is demonstrated with a specific and simple example. We now extend the discussion to complex problems which need to be dealt with by more complicated methods which are generally applicable. The cell dimensions in a rhombohedral lattice are $|\mathbf{a}|=|\mathbf{b}|=|\mathbf{c}|$ and $\alpha=\beta=\gamma$. For certain angles, different lattices are obtained as explained in "Appendix". The conventional $C P$ cell shown by Fig. 7 can be considered as a primitive rhombohedral cell. However, what we consider here will be an non-conventional I-centered $(r l)$ rhombohedral cell with axes $\mathbf{O} \mathbf{A}^{\prime}, \mathbf{O B}^{\prime}$ and $\mathbf{O} \mathbf{C}^{\prime}$ in Fig. 7 and a non-conventional primitive $(r P)$ rhombohedral cell with $\mathrm{O}^{\prime}$ removed from the cell. Both the non-conventional cells have the same unit cell parameters. $O B A^{\prime} C$ is the $(011)$ lattice plane and $A B C$ is the (111) lattice plane in these two rhombohedral lattices. The coordinates of the body center are $(1 / 2,1 / 2,1 / 2)$ in the non-conventional $l$-centered rhombohedral cell in Fig. 7. We can obtain $q(=2)$ for the lattice plane with Miller indices (111) by inserting these coordinates into from Eq. 14.

$$
\begin{aligned}
h x+k y+l z & =1 \times 1 / 2+1 \times 1 / 2+1 \times 1 / 2 \\
& =s+\frac{m}{q}=1+\frac{1}{2}
\end{aligned}
$$

For (011) plane in the $r l$ cell, $q=1$. Applying Eq. 27 or 59 in [39] or Eq. 29 in this paper, we obtain

$$
\begin{aligned}
\left|\mathbf{a}^{\prime}\right|=\mid \mathbf{O A} & =\left|\mathbf{b}^{\prime}\right|=|\mathbf{O B}|=\left|\mathbf{c}^{\prime}\right|=\left|\mathbf{O} \mathbf{C}^{\prime}\right|=\sqrt{2}|\mathbf{O B}| \\
\begin{aligned}
\alpha= & \frac{\pi}{3}
\end{aligned} & \\
d_{011}(r l) & =\frac{1}{q} d_{011}(r P) \\
& =\frac{1}{1} \sqrt{\frac{\left|\mathbf{a}^{\prime}\right|^{2}\left(1-3 \cos ^{2} \alpha+2 \cos ^{3} \alpha\right)}{\left(h^{2}+k^{2}+l^{2}\right) \sin ^{2} \alpha-2(h k+k l+l h)\left(\cos \alpha-\cos ^{2} \alpha\right)}} \\
& =\sqrt{\frac{2|\mathbf{O B}|^{2}\left(1-3 \times \frac{1}{4}+2 \times \frac{1}{8}\right)}{2 \times \frac{3}{4}-2 \times\left(\frac{1}{2}-\frac{1}{4}\right)}}=|\mathbf{O B}|=d_{100}(C P)
\end{aligned}
$$$$
d_{111}(r l)=\frac{1}{q} d_{111}(r P)
$$

$$
=\frac{1}{2} \sqrt{\frac{2|\mathbf{O B}|^{2}\left(1-3 \times \frac{1}{4}+2 \times \frac{1}{8}\right)}{3 \times \frac{3}{4}-2 \times 3\left(\frac{1}{2}-\frac{1}{4}\right)}}=\frac{\sqrt{3}|\mathbf{O B}|}{3}=d_{111}(C P)
$$


The same result as given by Eq. 49 and 50 is obtained from both the conventional simple cubic and the non-conventional body-centered rhombohedral lattices

$\frac{\rho\left(S_{111}, C P\right)}{\rho\left(S_{100}, C P\right)}=\frac{\rho\left(S_{111}, r l\right)}{\rho\left(S_{011}, r l\right)}=\frac{d_{111}(r l)}{d_{011}(r l)}=\frac{\sqrt{3}}{3}$

The bulk density of $r P$ lattice is reduced by a factor of 2 compared with that of $r$ lattice; thus, the density of every lattice plane in $r P$ lattice is reduced by a factor of $2 . q=1$ for all primitive lattices. Thus, from Eq. 54 the relative node density of (111) planes compared with that of (011) planes for $r$ lattice is reduced by a factor of 2 . This is because, by adding node $\mathrm{O}^{\prime}$ in the unit cell of $r P$ lattice, a new plane has been inserted into the middle of every two adjacent (111) planes but no new plane has been added to in the (011) planes. Issues like these cause difficulty in Fan's PD method and make additional considerations necessary.

The advantage of our method is that $d_{h k l}$ in Eq. 45 or 46 is still calculated from the original lattice, rhombohedral in this example, even though the cell for $V_{h k l}$ is no longer rhombohedral. The cell for $V_{h k l}$ is only conceptual, i.e., in the calculations, detailed information of the cell for $V_{h k l}$ is not needed, or to put it another way, the exact shape of $S_{h k l}$ does not need to be characterized even it is fully determined by $(h k l)$ in the rhombohedral lattice. It should be noted that $(h k l)$ in Eq. 45 or 46 are obtained from the original rhombohedral lattice but not from the lattice characterized by the cell for $V_{h k l}$. The cell for $V_{h k l}$ is just a parallelepiped in the rhombohedral lattice.

Since the volume of the unit cell for the non-conventional $r$ lattice from Fig. 7 is twice that of the conventional $C P$ lattice, (Eqs. 19-23) the plane area for the unit cell $S_{111}(r l)$ is twice that for the $C P\left[=S_{111}(C P)\right]$. A (111) plane from Fig. 7 is constructed in Fig. 9. A non-conventional non-primitive hexagonal lattice [26] $h P^{\prime}$ with axes $\mathbf{a}^{\prime \prime}, \mathbf{b}^{\prime \prime}$, and $\mathbf{c}^{\prime \prime}$ can be assigned to $C P$ (Figs. 7 and 9). The hexagonal crystal $\mathrm{BaFe}_{12} \mathrm{O}_{19}$ can be considered as being constructed from $A B C A$ packing in this way [26]. Instead of the rhombohedral lattice, the calculation can also be based on this hexagonal lattice.

$$
\begin{aligned}
\left|\mathbf{a}^{\prime \prime}\right| & =\sqrt{2}|\mathbf{O B}|,\left|\mathbf{b}^{\prime \prime}\right|=\sqrt{2}|\mathbf{O B}|,\left|\mathbf{c}^{\prime \prime}\right|=\sqrt{3}|\mathbf{O B}| \\
\alpha & =\beta=\frac{\pi}{2}, \gamma=\frac{2 \pi}{3}
\end{aligned}
$$

The relationship between the Miller indices for the $h P^{\prime}$ and $C P$ lattices is given by

$\left[\begin{array}{l}h\left(h P^{\prime}\right) \\ k\left(h P^{\prime}\right) \\ l\left(h P^{\prime}\right)\end{array}\right]=\left(\begin{array}{ccc}-1 & 1 & 0 \\ 0 & -1 & 1 \\ 1 & 1 & 1\end{array}\right)\left[\begin{array}{l}h(c P) \\ k(c P) \\ l(c P)\end{array}\right]$

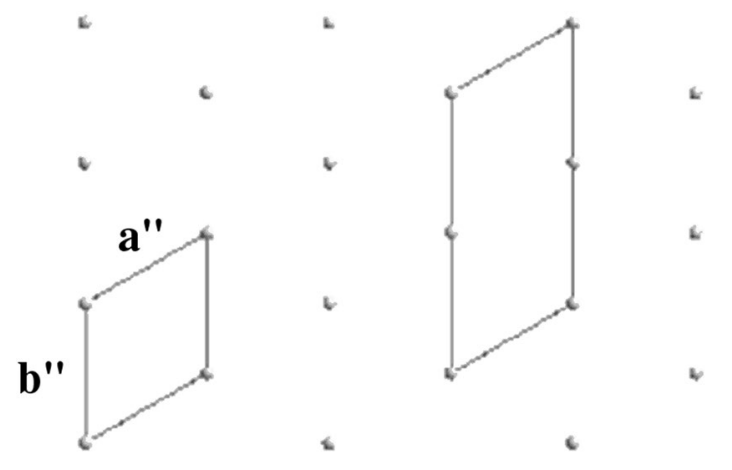

Fig. 9 A planar lattice with Miller indices (111) from a $c P$ lattice associated with Fig. 7. The planar unit cell with axes $\mathbf{A B}=\mathbf{a}^{\prime \prime}=\mathbf{b}-\mathbf{a}$ and $\mathbf{B C}=\left|\mathbf{b}^{\prime \prime}\right|=\mathbf{c}-\mathbf{b}$ contains one lattice node (Fig. 7). A non-conventional hexagonal lattice $\left(h P^{\prime}\right)$ with a threedimensional non-primitive unit cell can be chosen for the $C P$ lattice with $\left|\mathbf{O O}^{\prime \prime}\right|=\left|\mathbf{c}^{\prime \prime}\right|=|\mathbf{a}+\mathbf{b}+\mathbf{c}|=3 d_{111}(C P)$ from ABCA packing. $c^{\prime \prime}$ is perpendicular to plane (111). A non-conventional monoclinic three-dimensional lattice can also be constructed from the larger cell indicated. In a monoclinic axis, $\mathbf{b}$ is conventionally the unique axis perpendicular to $\mathbf{a}$ and $\mathbf{c}$, but here $\mathbf{c}^{\prime \prime}$ is in place of $\mathbf{b}$

The (100) planes in $C P$ are the $(\overline{1}, 0,1)$ planes in $h P^{\prime}$.

$\left(\begin{array}{ccc}-1 & 1 & 0 \\ 0 & -1 & 1 \\ 1 & 1 & 1\end{array}\right)\left(\begin{array}{l}1 \\ 0 \\ 0\end{array}\right)=\left(\begin{array}{c}-1 \\ 0 \\ 1\end{array}\right)$

If the origin of the $h P^{\prime}$ lattice is shifted from $O$ to $B$ in Fig. 7, the relationship between $h P^{\prime}$ and $C P$ will not change. Nodes $O^{\prime}(-1 / 3,1 / 3,2 / 3), C^{\prime}(-2 / 3,-1 / 3,1 / 3), A(-1,0$, $0), B^{\prime}(-2 / 3,2 / 3,1 / 3)$ are located on the plane with Miller indices $(\overline{1}, 0,1)$ and $n=1$ in Eq. 4 or 59 for the lattice plane in $h P^{\prime}$ lattice. Points $\mathrm{O}(-1 / 3,1 / 3,-1 / 3), \mathrm{B}(0,0,0), \mathrm{A}^{\prime}(1 / 3$, $2 / 3,1 / 3), C(0,1,0)$ are located on the plane with $n=0$.

$h^{\prime} x+k^{\prime} y+l^{\prime} z=-x+z=n$

The $(010)$ planes in $C P$ are the $(1, \overline{1}, 1)$ planes in $h P^{\prime}$.

$\left(\begin{array}{ccc}-1 & 1 & 0 \\ 0 & -1 & 1 \\ 1 & 1 & 1\end{array}\right)\left(\begin{array}{l}0 \\ 1 \\ 0\end{array}\right)=\left(\begin{array}{c}1 \\ -1 \\ 1\end{array}\right)$

Nodes $O(-1 / 3,1 / 3,-1 / 3), A(-1,0,0), C(0,1,0), B^{\prime}(-2 / 3$, $2 / 3,1 / 3)$ on the plane with $(1, \overline{1}, 1)$ and $n=-1$, and points $\mathrm{O}^{\prime}(-1 / 3,1 / 3,2 / 3), \mathrm{A}^{\prime}(1 / 3,2 / 3,1 / 3), \mathrm{B}(0,0,0), \mathrm{C}^{\prime}(-2 / 3$, $-1 / 3,1 / 3)$ at $n=0$.

$h^{\prime} x+k^{\prime} y+l^{\prime} z=x-y+z=n$ 
The (001) planes in $c P$ are the (011) planes in $h P^{\prime}$.

$\left(\begin{array}{ccc}-1 & 1 & 0 \\ 0 & -1 & 1 \\ 1 & 1 & 1\end{array}\right)\left(\begin{array}{l}0 \\ 0 \\ 1\end{array}\right)=\left(\begin{array}{l}0 \\ 1 \\ 1\end{array}\right)$

Nodes $O^{\prime}(-1 / 3,1 / 3,2 / 3), A^{\prime}(1 / 3,2 / 3,1 / 3), C(0,1,0), B^{\prime}(-2 / 3$, $2 / 3,1 / 3$ ) located on planes with $(011)$ and $n=1$, and point $O(-1 / 3,1 / 3,-1 / 3), A(-1,0,0), C^{\prime}(-2 / 3,-1 / 3,1 / 3), B(0,0$, 0 ) on planes with $n=0$.

$h^{\prime} x+k^{\prime} y+l^{\prime} z=y+z=n$

When $n$ is an integer, $q=1$. Thus, the interplanar spacing in each of the family above is given by Eq. 64 from Eqs. 23, 24 , and 46 in [39] with $\alpha=\beta=\pi / 2, \gamma=2 \pi / 3$.

$$
\begin{aligned}
d_{h^{\prime} k^{\prime \prime} \prime^{\prime}}\left(h P^{\prime}\right) & =\frac{1}{q} d_{h^{\prime} k^{\prime \prime} \prime^{\prime}}(h P) \\
& =\frac{\sin \gamma}{\sqrt{\frac{h^{\prime 2}}{\left|\mathbf{a}^{\prime \prime}\right|^{2}}+\frac{k^{\prime 2}}{\left|\mathbf{b}^{\prime \prime}\right|^{2}}+\frac{\left.\right|^{\prime 2} \sin ^{2} \gamma}{\left|\mathbf{c}^{\prime \prime}\right|^{2}}-\frac{2 h^{\prime} k^{\prime} \cos \gamma}{\left|\mathbf{a}^{\prime \prime}\right|\left|\mathbf{b}^{\prime \prime}\right|}}} \\
& =\frac{\sin \gamma}{\sqrt{\frac{h^{\prime 2}}{2|\mathbf{a}|^{2}}+\frac{k^{\prime 2}}{2|\mathbf{a}|^{2}}+\frac{3 l^{\prime 2} / 4}{3|\mathbf{a}|^{2}}-\frac{2 h^{\prime} k^{\prime}(-1 / 2)}{2|\mathbf{a}|^{2}}}} \\
& =\frac{1}{\sqrt{\frac{4}{3}\left(\frac{h^{\prime 2}+h^{\prime} k^{\prime}+k^{\prime 2}}{2|\mathbf{a}|^{2}}\right)+\frac{l^{\prime 2}}{3|\mathbf{a}|^{2}}}} \\
& =|\mathbf{a}|=d_{h k l}(c P)=\frac{|\mathbf{a}|}{\sqrt{h^{2}+k^{2}+l^{2}}}
\end{aligned}
$$

Equation 64 shows that the hexagonal lattice can be considered to be a special case of a monoclinic lattice (Eqs. 18 and 23 , or 35 , in [39]). The (111) planes in $C P$ are the (001) planes in $h P^{\prime}$ lattice.

$\left(\begin{array}{ccc}-1 & 1 & 0 \\ 0 & -1 & 1 \\ 1 & 1 & 1\end{array}\right)\left(\begin{array}{l}1 \\ 1 \\ 1\end{array}\right)=\left(\begin{array}{l}0 \\ 0 \\ 3\end{array}\right) \Rightarrow\left(\begin{array}{l}0 \\ 0 \\ 1\end{array}\right)$

From the coordinates of the lattice nodes and Eq. 66, we obtain $q=3$ for (001) planes in $h P^{\prime}$ lattice and their interplanar spacing.

$h^{\prime} x+k^{\prime} y+l^{\prime} z=z=s+\frac{m}{q}$

$$
\begin{aligned}
d_{001}\left(h P^{\prime}\right) & =\frac{1}{9} d_{001}(h P)=\frac{1}{q} d_{h^{\prime} k^{\prime} l^{\prime}}(h P) \\
& =\frac{1}{3} \frac{1}{\sqrt{\frac{4}{3}\left(\frac{h^{\prime 2}+h^{\prime} k^{\prime}+k^{\prime 2}}{2|\mathbf{a}|^{2}}\right)+\frac{l^{\prime 2}}{3|\mathbf{a}|^{2}}}}=\frac{\sqrt{3}}{3}|\mathbf{a}| \\
& =\frac{1}{3} \frac{\sin \gamma}{\sqrt{\frac{h^{\prime 2}}{\left|\mathbf{a}^{\prime}\right|^{2}}+\frac{k^{\prime 2}}{\left|\mathbf{b}^{\prime}\right|^{2}}+\frac{l^{\prime 2} \sin ^{2} \gamma}{\left|\mathbf{c}^{\prime}\right|^{2}}-\frac{2 h^{\prime} k^{\prime} \cos \gamma}{\left|\mathbf{a}^{\prime}\right|\left|\mathbf{b}^{\prime}\right|}}} \\
& =\frac{1}{3} \frac{\sin \gamma}{\sqrt{\frac{h^{\prime 2}}{2|\mathbf{a}|^{2}}+\frac{k^{\prime 2}}{2|\mathbf{a}|^{2}}+\frac{3 \prime^{\prime 2} / 4}{3|\mathbf{a}|^{2}}-\frac{2 h^{\prime} k^{\prime}(-1 / 2)}{2|\mathbf{a}|^{2}}}} \\
& =\frac{1}{3} \frac{\sqrt{3} / 2}{\sqrt{\frac{0}{2|\mathbf{a}|^{2}}+\frac{0}{2|\mathbf{a}|^{2}}+\frac{3 / 4}{3|\mathbf{a}|^{2}}+\frac{0}{2|\mathbf{a}|^{2}}}} \\
& =\frac{\sqrt{3}}{3}|\mathbf{O B}|=\frac{1}{3}\left|\mathbf{O O} \mathbf{O}^{\prime}\right| \\
& =d_{111}(c P)=d_{h k \mid}(c P)
\end{aligned}
$$

The same result as Eq. 49 is still obtained from Eqs. 64 and 67. Similar calculations with a triclinic or a larger monoclinic lattice can be based on the planar unit cell with two lattice nodes shown in Fig. 9. From these calculations, it can be seen that the modification of the definition of Miller indices with respect to an individual plane [1] has no particular merit in the calculations of interplanar spacing. In calculation, the individual lattice plane is used to obtain the Miller indices of the redefinition while the individual plane is only considered when it is needed with the conventional definition of Miller indices. The difference here between the conventional definition and the modification is found only in the sequence of calculation procedures.

\subsubsection{The face-centered cubic lattice}

$\triangle A B C$ can represent a lattice plane in the family of (111) planes for the primitive rhombohedral $h R$ lattice in Fig. 6 . The lattice plane that passes through $B$ and is perpendicular to $\mathbf{O} \mathbf{C}^{\prime}$ is one of the family of (110) planes in the $h R$ lattice. The relative node density for the two planes is

$$
\begin{aligned}
\frac{\rho\left(S_{111}, h R\right)}{\rho\left(S_{110}, h R\right)} & =\frac{\frac{1 / 2}{8}\left|\mathbf{O A}^{\prime}\right|^{2}}{2 /\left|\mathbf{O A}^{\prime}\right|^{2}}=\frac{d_{111}(h R)}{d_{110}(h R)}=\frac{\frac{\sqrt{3}}{3}\left|\mathbf{O A}^{\prime}\right|}{\frac{1}{2}\left|\mathbf{O A}^{\prime}\right|} \\
& =\frac{2 \sqrt{3}}{3}=N^{\prime}
\end{aligned}
$$


The result agrees with that obtained from the same nonprimitive $c$ F lattice (Fig. 6) where $\triangle A^{\prime} B^{\prime} C^{\prime}$ has been taken as the representative lattice plane for the (111) family.

$$
\begin{aligned}
\frac{\rho\left(S_{111}, C F\right)}{\rho\left(S_{100}, C F\right)} & =\frac{\frac{2}{\frac{\sqrt{3}}{2}\left|\mathbf{O A}^{\prime}\right|^{2}}}{2 /\left|\mathbf{O} \mathbf{A}^{\prime}\right|^{2}}=\frac{d_{111}(c F)}{d_{100}(C F)}=\frac{\frac{\sqrt{3}}{3}\left|\mathbf{O A}^{\prime}\right|}{\frac{1}{2}\left|\mathbf{O A}^{\prime}\right|} \\
& =\frac{2 \sqrt{3}}{3}=N^{\prime}
\end{aligned}
$$

The nodes on the lattice plane with Miller indices (100) are denser than those on the plane with Miller indices (111) for the $c P$ lattice in Fig. 7 as indicated by Eq. 50 . However this latter (111) plane is denser than the (110) plane (Fig. 6) for the primitive lattice $h R$ as indicated by Eq. 68 and the (100) plane for the non-primitive $c F$ lattice as indicated by Eq. 69 . The faces of a crystal are usually found to be parallel to lattice planes with a high density of lattice nodes. For example the prominent $\{111\}$ faces to be found on $C F$ crystals of $\mathrm{Fe}_{3} \mathrm{O}_{4}$ are shown by Fig. 14b in [57]. As shown from Figs. 6 and 9 , the (111) planes in $C F$ crystal are the (001) planes in the hexagonal $\mathrm{BaFe}_{12} \mathrm{O}_{19}$ crystal. The prominent (001) faces in $\mathrm{BaFe}_{12} \mathrm{O}_{19}$ crystal can be identified from Fig. 14a in [57].

\subsection{A summary of Sect. 3}

The calculations of node density of lattice plane from a recent paper [4] have been reanalyzed with simple principles which leads to a different perspective on the conventional definition of Miller indices. The basic concept that all parallel lattice planes in a family are equivalent whether the lattice is primitive or not is imbedded in the definition. By improperly generalizing from specific calculations, Fan [4] claimed that the node densities for lattice planes could be different within a family of parallel planes characterized by the same set of Miller indices. The problem with this generalization is caused by confusing the concepts of lattice nodes in a lattice and atoms in a crystal. By confusing these two different concepts, Fan [4] calculated his $P D$ numbers from Eq. 31 with $r=m / q$ specifying that $m$ and $q$ are both integers.

Fan claimed that some planes with $m=0,1,2, \ldots,(q-1)$ could be missing. In fact any of the plane in the set is not allowed to be missing in a lattice and $r$ can be a real number for atoms in a crystal. There is also an incurable problem with Fan's $P D$ number method and the problem has been illustrated with examples in Sect. 3.2. These problems can be avoided by converting the non-primitive lattice to a primitive lattice and then carrying out the calculations. In this way, the calculations of $P D$ numbers in [4] can be avoided since there is only one lattice point in a primitive unit cell. More importantly by choosing different primitive unit cells associated with different Miller indices in a primitive lattice, a method is developed to work out the relationship of lattice node densities between planes with different Miller indices in Sect. 3.4. The relative lattice node density for planes is directly related with interplanar spacing. The method here is developed with a primitive lattice but it can be used for a non-primitive lattice which has been shown with a hexagonal lattice from Fig. 9.

Some aspects of the conventional definition of Miller indices have been misunderstood which have led to inaccurate modifications [1]. Unfortunately, this type of mistake where researchers attempt an unnecessary and inaccurate "improvement" of a well-used theory is not uncommon. The discussions above show the value of fundamental theory played in today's academic world.

\section{Interplanar spacing in primitive and non-primitive lattices and in a crystal}

In another paper, [5] it was claimed that a new method for calculating interplanar spacing in lattices and crystals had been established. By generalizing the results of calculations on specially located atoms in a crystal, the author found parallel lattice planes can be unevenly distributed. It was claimed that the correctness of the method and conclusions had been ensured by correcting the problems that occur in calculation of powder patterns presented on JCPDS-ICDD cards. The issue is related with interplanar spacing [58] and the definition of Miller indices. However, this claimed new method is just a combination of the improper redefinition of Miller indices and some correct methods developed long ago and thus leads to confusions [22, 39].

\subsection{The equivalence for interplanar spacing in the same lattice expressed as primitive and non-primitive}

The equations for planes with any Miller indices $(h k l)$ in a primitive lattice are

$$
\begin{gathered}
h x+k y+l z=-1 \\
h x+k y+l z=0 \\
h x+k y+l z=1 \\
h x+k y+l z=2 \\
\ldots
\end{gathered}
$$




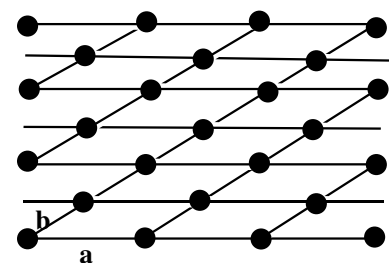

(a)

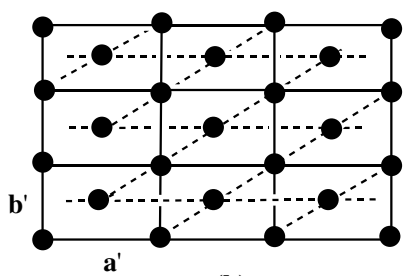

(b)
Fig. 10 The same lattice can be expressed as either a primitive lattice (a) or a non-primitive centered lattice (b). The black dots represent lattice nodes. The axes of the two lattices are related by $\mathbf{a}^{\prime}=\mathbf{a}$ and $\mathbf{b}^{\prime}=2 \mathbf{b}-\mathbf{a}$. Lattice lines represent lattice planes perpendicular to the paper. The interplanar spacing in the lattice planes cannot be changed by changing the representation from primitive to nonprimitive

Equations $70-73$ are summarized by Eq. 4 or 31 . Any set of parallel planes in a primitive lattice are represented by these equations. Thus lattice planes represented by such as Eq. 74 cannot exist because the principles embedded in the definition of Miller indices.

$h x+k y+l z=\sqrt{3}-1$

What is more, in a primitive lattice there cannot exist an additional intermediate lattice plane represented by such as Eq. 75 between the planes represented by Eqs. 71 and 72.

$h x+k y+l z=0.3$

Equations 70-73 and their implications to exclude Eqs. 74 and 75 are consistent with the conventional definition of Miller indices for a lattice. Thus, the spacing between any adjacent planes in the series are the same in a primitive lattice, i.e., the planes are evenly distributed. There cannot be uneven spacing in a set of parallel lattice planes since the family of parallel planes can be constructed by any one of these planes and a translation operation. Fig. 10 shows another aspects of Fig. 5 .

It is clear that the Miller indices in a primitive lattice may not be the same as the Miller indices in a non-primitive lattice when they refer to the same set of parallel planes. To distinguish the nomenclature here, we use $(h k l)$ and $\left(h^{\prime} k^{\prime} l^{\prime}\right)$ to represent planes in primitive and non-primitive lattices, respectively. In [5], the author made no distinction between the non-primitive lattice and the crystal because it was assumed (wrongly) that the conclusions for the former would also apply to the latter. However, the conclusions cannot be justified. In a primitive lattice, the interplanar spacing $d_{h k l}$ can be calculated by the wellknown formulae developed long ago $[37,39,58]$. These formulae can also be used for non-primitive lattices where the interplanar spacing is given by $(1 / q) d_{h^{\prime} k^{\prime} l^{\prime}}$ which is calculated from the traditional method with reference to the conventional Miller indices and axes in a non-primitive lattice. The relevant calculations have also been demonstrated especially in Sect. 3.4. If the interplanar spacing for a non-primitive lattice could be calculated from $(m / q) d_{h^{\prime} k^{\prime} l^{\prime}}$ as claimed in [5], then some of the lattice planes would have been missed and the lattice planes would be distributed unevenly which cannot happen [39]. The wrong conclusions were obtained by calculations using positions which were not lattice nodes. The conclusions in [5] are also incorrect when applied to atoms in a crystal as will be demonstrated in the following sections.

\subsection{An illustrative demonstration of the correct calculations}

The conclusions in [5] were obtained by generalizing the results from specific calculated examples: e.g. "... For the seven centered lattices of the 14 Bravais lattices, one unit cell contains more than one lattice point, and those lattice points that are not located at the corners of the unit cell can be called 'additional lattice points' ... and those atoms that are not located at the corners of the unit cell can be called 'additional atoms".' [4] It was also stated that $P D^{\prime \prime}$... is the scalar product of an additional atom at $(\mathrm{x}, \mathrm{y}$, $\mathrm{z}$ ) and any (hkl) plane passing through that additional atom, and it can be used for calculating the interplanar spacing of the (hkl) plane," "it can be seen that for an h.c.p. crystal there are six possible kinds of interplanar spacing: $d_{h^{\prime} k^{\prime} \nu^{\prime}} / 6, d_{h^{\prime} k^{\prime} \nu^{\prime}} / 3, d_{h^{\prime} k^{\prime} \prime^{\prime}} / 2,2 d_{h^{\prime} k^{\prime} \nu^{\prime}} / 3,5 d_{h^{\prime} k^{\prime} l^{\prime}} / 6$ and $d_{h^{\prime} k^{\prime} l^{\prime}}$. Obviously, for an additional plane that is not located in the middle of two adjacent atom planes, there are two kinds of interplanar spacing which are not equal but complementary: $d_{h^{\prime} k^{\prime} \prime^{\prime}} / 3$ and $2 d_{h^{\prime} k^{\prime \prime} \prime^{\prime}} / 3$, or $d_{h^{\prime} k^{\prime \prime} \prime^{\prime}} / 6$ and $5 d_{h^{\prime} k^{\prime \prime} \prime^{\prime}} / 6$." $[5]$ The papers $[4,5]$ include many descriptions which confuse lattice nodes with atoms in crystal, an error that also occurred in the redefinition of Miller indices. For example, atoms in a crystal can occupy any possible position in a unit cell while a lattice node is restricted to the center or corner of a Bravais lattice.

Although two types of positions are distinguished by "additional lattice points" and "additional atoms," results from calculations do not sufficiently distinguish these two terms. Only the center and corner in Bravais are standard positions possible for lattice node. If the "additional atoms" occupy the positions of lattice nodes in a lattice cell, then there are not so many possibilities for the spacing of parallel lattice planes since the planes are uniformly distributed which is required by Miller indices. If the planes can be distributed unevenly with various different possible spacings among the six values given above, then (222) is possible for Miller indices of these parallel planes as claimed 


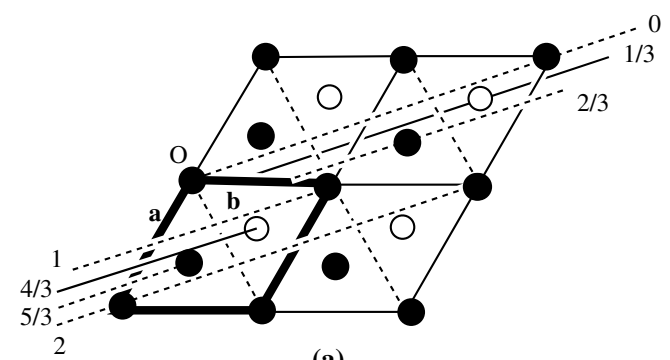

(a)

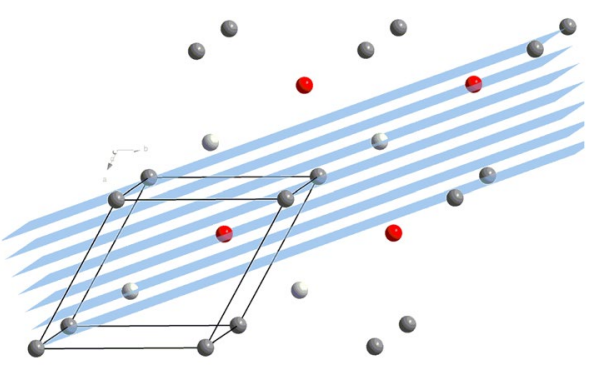

(b)

Fig. 11 a The missing lattice planes caused by the missing lattice points. The diagram shows the ab projection of atoms (black dots) in hexagonal close-packing. Open circles represent the missing lattice points. A number of planes with Miller indices (210) are indicated along with the value of $r$ in Eq. 31. The distance from $\mathrm{O}$ to a plane is $d=r d_{h^{\prime} k^{\prime} I^{\prime}}$. The planes presented are indicated by dashed lines. The planes are not distributed evenly since the planes with $r$ $=1 / 3$ and $4 / 3$ represented by solid lines are missing. $\mathbf{b}$ The perspective view for that represented by (a). Black, gray, and red balls indicate three different kinds of points

wrongly in [1, 3]. If the "additional atoms" occupy arbitrary positions in a crystal unit cell, then there are an infinite number of possibilities for the uneven distributions of parallel crystal planes all with the same $(h k l)$. To explain the errors in the calculations and the generalization in [5], we first provide calculations that are more relevant. Fig. 11a shows a hexagonal close-packing model $[26,27]$. The distance from point $O$ to the plane passing through the atom with coordinates $(x, y, z)$ and with Miller indices (210) can be calculated as follows. For an atom positioned at $(0,1$, $0)$, we obtain

$$
\begin{aligned}
d_{h^{\prime} k^{\prime} \prime^{\prime}}(x y z) & =d_{210}(010) \\
& =(x \mathbf{a}+y \mathbf{b}+z \mathbf{c})\left[\left(h \mathbf{a}^{*}+k \mathbf{b}^{*}+l \mathbf{c}^{*}\right) d_{h^{\prime} k^{\prime \prime} l^{\prime}}\right] \\
& =(h x+k y+l z) d_{h^{\prime} k^{\prime} l^{\prime}} \\
& =(0+1+0) d_{210}=d_{210}
\end{aligned}
$$

For an atom positioned at $(1,0,0)$

$$
\begin{aligned}
d_{h^{\prime} k^{\prime} l^{\prime}}(x y z) & =d_{210}(100) \\
& =(h x+k y+l z) d_{h^{\prime} k^{\prime} l^{\prime}} \\
& =(1 \times 2+0 \times 1+0 \times 0) d_{210}=2 d_{210}
\end{aligned}
$$

For an atom positioned at $(2 / 3,1 / 3,0)$

$$
\begin{aligned}
d_{h^{\prime} k^{\prime} l^{\prime}}(x y z) & =d_{210}(2 / 3,1 / 3,0) \\
& =(h x+k y+l z) d_{h^{\prime} k^{\prime} l^{\prime}} \\
& =\left(\frac{2}{3} \times 2+\frac{1}{3} \times 1+0 \times 0\right) d_{210}=\frac{5}{3} d_{210}
\end{aligned}
$$

For an atom positioned at $(-1 / 3,4 / 3,0)$

$$
\begin{aligned}
d_{h^{\prime} k^{\prime} \prime^{\prime}}(x y z) & =d_{210}(-1 / 3,4 / 3,0) \\
& =(h x+k y+l z) d_{\left.h^{\prime} k^{\prime}\right|^{\prime}} \\
& =\left(-\frac{1}{3} \times 2+\frac{4}{3} \times 1+0 \times 0\right) d_{210}=\frac{2}{3} d_{210}
\end{aligned}
$$

The above planes are indicated in Fig. 11a by dashed lines. The missing planes involving the missing atoms (open circles) are indicated by solid lines. For the missing atom at $(1 / 3,2 / 3,0)$

$$
\begin{aligned}
d_{h^{\prime} k^{\prime \prime} \prime}(x y z) & =d_{210}(1 / 3,2 / 3,0) \\
& =(h x+k y+l z) d_{h^{\prime} k^{\prime} I^{\prime}} \\
& =\left(\frac{1}{3} \times 2+\frac{2}{3} \times 1+0 \times 0\right) d_{210}=\frac{4}{3} d_{210}
\end{aligned}
$$

For the missing atom at $(-2 / 3,5 / 3,0)$

$$
\begin{aligned}
d_{h^{\prime} k^{\prime} l^{\prime}}(x y z) & =d_{210}(-2 / 3,5 / 3,0) \\
& =(h x+k y+l z) d_{h^{\prime} k^{\prime} l^{\prime}} \\
& =\left(-\frac{2}{3} \times 2+\frac{5}{3} \times 1+0 \times 0\right) d_{210}=\frac{1}{3} d_{210}
\end{aligned}
$$

If there were no missing atoms, Fig. 11 would provide a lattice in which no plane is missing and all the planes would be evenly distributed. However, for hexagonal close-packing, some atoms are missing and some planes are missing and not all the black points in Fig. 11a are lattice nodes. Thus, the planes are not evenly distributed and the spacing between planes are not equal.

When there are no missing points, Fig. 11 represents a complete non-primitive lattice because the axes $\mathbf{a}$ and b are long and some of the lattice nodes must be represented by fractional coordinates. However, the result of the uneven distribution of planes calculated above from missing points does not prove that lattice planes can be unevenly distributed since parts of the remaining nodes will no longer be the lattice nodes. Lattice nodes and atoms in a crystal represent different concepts. As pointed out from Sect. 2, the diamond structure can be described by a face-centered cubic non-primitive unit cell with four lattice nodes but eight carbon atoms. It can also be described by 
a rhombohedral unit cell with one lattice node or 2 carbon atoms. For a lattice, whether it is primitive or non-primitive, the spacing between adjacent planes is always the same for a set of parallel planes with the specific Miller indices. Thus the interplanar spacing for a primitive lattice is $d_{h k l}$ and the interplanar spacing for a non-primitive lattice is $(1 / q) d_{h^{\prime} k^{\prime} l^{\prime}}$, but not a specific $(m / q) d_{h^{\prime} k^{\prime \prime} l^{\prime}}$ as claimed in [5]. The same environment for every lattice node, the existence of translation operations, the same node density for every plane in a parallel set, the even distribution of lattice planes, the law of rational indices, and the conventional definition of Miller indices form a consistent theoretical system.

\subsection{The difference between lattice nodes and atoms in a crystal}

Since not all the atoms in a hexagonal close-packing cell are positioned as lattice nodes, it might appear that the conclusions claimed in [5] were correct for an atom in a crystal even though they are problematic with a lattice. The uneven interplanar spacing does indeed originate from the values $(m / q) d_{h^{\prime} k^{\prime} l^{\prime}}$ available in Fig. 11 . But the result is not valid for lattice planes. Although the planes in a crystal can be missing and the interplanar spacing can take several different values, the extra atom at $(2 / 3,1 / 3$, 0 ) in the hexagonal close-packing is located at a special position. An atom in a crystal can occupy any position in the unit cell relative to the lattice node and coordinates of the atom can have any values with respect to its relative position in a crystal. Indeed, the value of $r$ in Eq. 31 can be $\sqrt{3}-1$. Thus the distance between adjacent planes in a crystal is $r d_{h^{\prime} k^{\prime} \prime}$ where $r$ is a real number instead of $r=m / q$, a rational number, claimed in [5].

It was claimed in [5] that results from the article could help solving the problems that occurred in JCPDS-ICDD cards, and this was used to show the effective and correctness of its method. The diffraction intensities are calculated from structure factors in which the interplanar distances are indirectly involved as shown by Eqs. 8 and 9. To account for the systematic absences of reflections within the diffraction pattern, it is necessary to calculate structure factors from the appropriate equations for the space group and atomic positions in which the interplanar distances or unit cell dimensions have already imbedded. The interplanar distances in the JCPDS-ICDD cards are not calculated from this "new method" claimed by [5] but from the formula available for over 50 years before this "new method" was presented. The "new method" is irrelevant for the problems mentioned in the paper for diffraction peak assignments. The conventional method for calculating interplanar spacing in a crystal works satisfactorily for indexing diffraction peaks.

\subsection{Is the method used in [5] novel?}

It was claimed in [5] that the method used was new. It was developed using the erroneous Eq. 4 in that paper which is included in present paper as Eq. 14. The correct form (Eq. 4 or 31 in the present paper) of this equation has been previously studied theoretically as long ago as 1957 by Deas and Hamill [59] who proved that any lattice plane can be expressed by Eq. 4 and conversely, any equation such as Eq. 4 with $n$ as an integer must be a lattice plane. This result implies that any lattice plane cannot be missing and the lattice planes must be evenly spaced.

Earlier still in 1955, Jaswon and Dove [60] developed Eq. 82 for an atom in a crystal where $\delta$ is any real number.

$h x+k y+l z=n+\delta$

The more correct Eq. 82 should be the basis of the method used in [5]. Thus, the method claimed in [5] cannot be claimed as new.

\subsection{The conventional calculation method for interplanar spacing}

It is said in [5] that "... the principle and method for calculating these correction coefficients have not been reported ..." for non-primitive lattice. This statement is not exactly true. There is previous literature $[43,59,60]$ concerning interplanar spacing and here we specifically consider the main ideas of Jaswon and Dove [60] in 1955 who dealt with the interplanar spacing in non-primitive lattices in two different ways:

(1) by transforming the coordinate system of the primitive lattice to obtain a non-primitive lattice representation. For example when dealing with the lattice planes with Miller indices $(h k l)$ in primitive lattice, they transformed the axes $\mathbf{a}, \mathbf{b}, \mathbf{c}$ in the primitive lattice to $\mathbf{a}^{\prime}, \mathbf{b}^{\prime}, \mathbf{c}^{\prime}$ where

$\mathbf{a}^{\prime}=l \mathbf{a}-h \mathbf{c}$

$\mathbf{b}^{\prime}=/ \mathbf{b}-k \mathbf{c}$

$\mathbf{c}^{\prime}=\mathbf{b}^{\prime} \times \mathbf{c}^{\prime}$

The three points $(0,0,0),(I, 0,-h)$, and $(0, I,-k)$ in the primitive lattice are situated on the plane described by Eq. 71 . Thus, $\mathbf{a}^{\prime}$ and $\mathbf{b}^{\prime}$ are on the plane represented by Eq. 71 and $\mathbf{c}^{\prime}$ is perpendicular to $\mathbf{a}^{\prime}$ and $\mathbf{b}^{\prime}$. The lattice formed by $\mathbf{a}^{\prime}$, $\mathbf{b}^{\prime}$, and $\mathbf{c}^{\prime}$ can be a non-primitive lattice. This is the principle they used to deal with interplanar spacing in a nonprimitive lattice.

(2) by translating a lattice node in the original lattice to a special position so that the original and the newly generated lattices become two penetrating lattices which when 
combined provide a crystal structure. If the lattice node in a primitive cubic lattice is transformed to the body center, then the two penetrating lattices form a body-centered non-primitive cubic lattice.

A lattice node may represent many atoms since the lattice node represents a structural unit (motif). For an atom in a crystal, Jaswon and Dove dealt with the matter by translating the lattice nodes in the primitive lattice to form an interpenetrating secondary structure. In the calculation only the relative position of the atom (described by real numbers) with reference to the lattice node is important. In Fig. 11 it is shown that a lattice point can be translated to a special position but does not give rise to another lattice point.

\subsection{A summary of Sect. 4}

Contrary to previous claims [5], the interplanar spacing $d_{h k l}$ in a primitive lattice can be correctly calculated with formulae developed long ago. The calculations can be easily extended to include non-primitive lattices since there are not many non-primitive Bravais lattices for each type of primitive lattice. The formula for a nonprimitive lattice is $(1 / q) d_{h^{\prime} k^{\prime} \mid}$ where $d_{h^{\prime} k^{\prime} l^{\prime}}$ is calculated with respect to cell parameters of the non-primitive lattice by the conventional method developed long ago. The interplanar spacing between adjacent parallel lattice planes in a family is always the same no matter whether the lattice is expressed as a primitive or a non-primitive lattice. The interplanar spacing between crystal planes can be unequal and the value is calculated by $r d_{h^{\prime} k^{\prime} l^{\prime}}$ where $r$ is a real number which not limited to the rational number $m / q$.

\section{Conclusions}

Experimental research is concentrated on reporting new data and new phenomena. By contrast a review provides new insights on published results often by emphasizing fundamental theory. This present paper is concerned with a specific topic from published papers $[1-5,59,60]$ which relate to the long existing fundamental properties of crystal lattices associated with the conventional definition of Miller indices, i.e., all the nodes in a lattice are equivalent as are all parallel lattice planes in a family. It is noteworthy that despite the fact that the recent papers [1-5] concerned with lattice planes have presented debatable procedures, their contents have not till now been challenged, a fact which highlights the somewhat indifferent attitude in modern research regarding fundamental principles. By reviewing the published results, a new method for calculating relative lattice planar density has been developed in Sect. 3.4, which is rooted on the fact that any form of unit for a lattice, conventional or non-conventional, is a truthful representation of the whole lattice.

Much scientific research is concerned with explaining novel experimental results. However, many of the puzzling results and phenomena published can in fact be resolved by involving simple long-standing principles [18]. For that reason, work evaluating and expanding these published data or results is extremely valuable especially since there are much published data not yet fully explained. However, it must be agreed that such work is currently considered to be outmoded and of a low level since the solution usually stems from rudimentary principles. The purpose of this work is not only to provide clarity on parts of basic crystallography but also to that research based on fundamental theory is still relevant in modern research and needed in comprehending published materials and in consolidating work already published, or in first hand research. "The flashiest" and "eye-catching" work can be "judged by the impact factor" of the journal in which it is published [16]. However, serious research providing fundamental understanding from rudimentary principles judged by its sustainability in scientific history might be the criterion for the best work.

Acknowledgements This work was supported by Foundation of Liaoning Province Education Administration (LZD201902).

\section{Compliance with ethical standards}

Conflicts of interest The authors declare that they have no conflict of interest.

\section{Appendix: Special cases for a rhombohedral lattice}

In assigning a conventional Bravais lattice, it is often noted that a rhombohedral lattice has cell parameters that meet the conditions $|\mathbf{a}|=|\mathbf{b}|=|\mathbf{c}|$ and $\alpha=\beta=\gamma$. However there are special cases when the angles take particular values. If the angles are $120^{\circ}$ then these parameters characterize a two-dimensional hexagonal lattice; if the angles are $60^{\circ}, 90^{\circ}$, or $109^{\circ} 28^{\prime}$, then the lattice will be cubic. This is obvious when the angles are $90^{\circ}$ as shown in Figs. 6 and 7.

When the angles are $109^{\circ} 28^{\prime}$ (Eq. 86 from cosine rule for triangle) then the lattice is body-centered cubic $\mathrm{Cl}$ as shown in Fig. 12. Layer $I_{1} I_{2} I_{3}$ is in the middle between layer $A B C$ and its parallel layer containing $O^{\prime}$. Their parallel layer containing $O$ is in the middle between layers $A B C$ and $A^{\prime} B^{\prime} C^{\prime}$. Interplanar spacing between these adjacent 


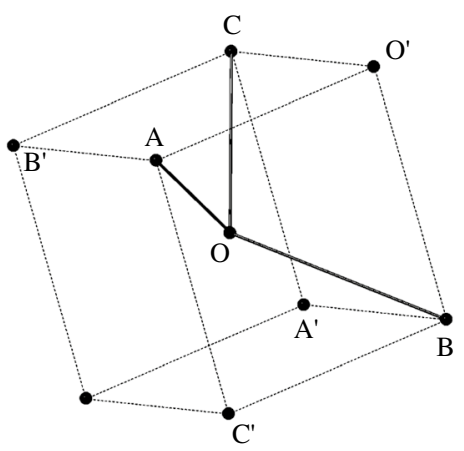

(a)

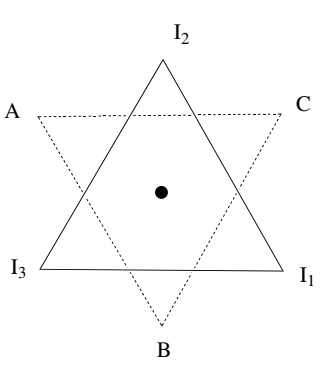

(b)

Fig. 12 a $A$ rhombohedral lattice with axes $\mathbf{O A}, \mathbf{O B}$, and $\mathbf{O C}$ and angle $109^{\circ} 28^{\prime}$ between them can be a body-centered cubic cell. $|\mathbf{O A}|=\frac{\sqrt{3}}{2}\left|\mathbf{A} \mathbf{O}^{\prime}\right|$ and $|\mathbf{A C}|=\sqrt{2}\left|\mathbf{A} \mathbf{O}^{\prime}\right|$. The body centers $I_{1}, I_{2}$, and $I_{3}$ at the opposite side of $O$ from lines $B C, A C$, and $A B$ are indicated in (b) where the dot represents $\mathrm{O}$ or $\mathrm{O}^{\prime}, \mathrm{O}$ being below layer $\mathrm{ABC}$ and

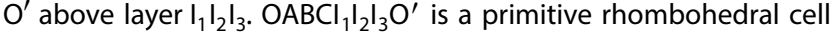
for this $\mathrm{Cl}$ lattice. There are four layers with Miller indices (111) from $\mathrm{O}$ to $\mathrm{O}^{\prime}$, two of which pass through $\mathrm{O}$ and $\mathrm{O}^{\prime}$, while the other two are layers $A B C$ and $I_{1} I_{2} I_{3}$

parallel layers is equal since the layers are evenly distributed in the lattice.

$\cos \angle A O C=\frac{|\mathbf{O A}|^{2}+|\mathbf{O C}|^{2}-|\mathbf{A C}|^{2}}{2|\mathbf{O A}||\mathbf{O C}|}=-\frac{1}{3}$

When $|\mathbf{O A}|=|\mathbf{O B}|=|\mathbf{O C}|=|\mathbf{A B}|$ in Fig. 6 and $\left|\mathbf{O A}^{\prime}\right|=\left|\mathbf{O B}^{\prime}\right|=\left|\mathbf{O C}^{\prime}\right|=\left|\mathbf{A}^{\prime} \mathbf{B}^{\prime}\right|$ in Fig. $7, \alpha=\beta=\gamma=60^{\circ}$. The lattices should be assigned to face-centered cubic $C F$ in Fig. 6 and to simple cubic $C P$ in Fig. 7.

In Fig. $6\left|\mathbf{A}^{\prime} \mathbf{B}^{\prime}\right|=\sqrt{2}\left|\mathbf{A}^{\prime} \mathbf{O}\right|$, thus $\angle A^{\prime} O B^{\prime}=90^{\circ}$. Another helpful perspective can be obtained from Fig. 13. The $A B C$ plane in Fig. 6 is shown in Fig. 13a. It can be proved from the perspective of Fig. 13 that the angles between $\mathbf{O A}^{\prime}$ and $\mathbf{O B}^{\prime}, \mathbf{O B}^{\prime}$ and $\mathbf{O C}^{\prime}$, and $\mathbf{O C}^{\prime}$ and $\mathbf{O A}^{\prime}$ are $90^{\circ}$.

Six equal spheres are close packed in a layer shown by Fig. 13a. $d$ is the diameter of the sphere. If another sphere $\mathrm{O}$ is placed on the top of spheres $\mathrm{A}, \mathrm{B}$, and $\mathrm{C}$, the same positions indicated in Fig. 6 are obtained. These positions have been redrawn in Fig. 13b.
From Fig. $13 \mathrm{~b}, \mathrm{ABCO}$ is a regular tetrahedron, thus, $|\mathbf{C E}|=|\mathbf{O E}|,|\mathbf{D E}|=|\mathbf{C E}| / 3=|\mathbf{O E}| / 3,\left|\mathbf{D A}^{\prime}\right|=|\mathbf{C E}|$ $+|\mathbf{D E}|=|\mathbf{O E}|+|\mathbf{O E}| / 3 ;|\mathbf{O A}|=|\mathbf{A B}|=d,|\mathbf{A E}|=|\mathbf{A B}| / 2 \mid$ $=d / 2,|\mathbf{O E}|^{2}=|\mathbf{O A}|^{2}-|\mathbf{A E}|^{2}=|\mathbf{O A}|^{2}-(|\mathbf{A B}| / 2)^{2}=$ $|\mathbf{O A}|^{2}-(|\mathbf{O A}| / 2)^{2}$. We obtain the length of $\mathbf{O A}^{\prime}$ from these relationships as shown in Eq.87.

$$
\begin{aligned}
\left|\mathbf{O A}^{\prime}\right| & =\sqrt{|\mathbf{O D}|^{2}+\left.|\mathbf{D A}|^{\prime}\right|^{2}} \\
& =\sqrt{\left(|\mathbf{O E}|^{2}-|\mathbf{D E}|^{2}\right)+(|\mathbf{C E}|+|\mathbf{D E}|)^{2}} \\
& =\sqrt{\left[|\mathbf{O E}|^{2}-\left(\frac{1}{3}|\mathbf{C E}|\right)^{2}\right]+\left(|\mathbf{C E}|+\frac{1}{3}|\mathbf{C E}|\right)^{2}} \\
& =\sqrt{\left.\left[|\mathbf{O E}|^{2}-\left(\frac{1}{3}|\mathbf{O E}|\right)^{2}\right]+\left[\left(1+\frac{1}{3}\right)|\mathbf{O E}|\right)\right]^{2}} \\
& =\sqrt{\frac{3 \times 8}{9}}|\mathbf{O E}| \\
& =\sqrt{\frac{3 \times 8}{9}} \sqrt{|\mathbf{O A}|^{2}-\left(\frac{1}{2}|\mathbf{O A}|\right)^{2}} \\
& =\sqrt{\frac{3 \times 8}{9}} \sqrt{\frac{3}{4}}|\mathbf{A B}|=\sqrt{2} d
\end{aligned}
$$

The angles between $\mathbf{O A}^{\prime}$ and $\mathbf{O B}^{\prime}, \mathbf{O B}^{\prime}$ and $\mathbf{O C}^{\prime}$, and $\mathbf{O C}^{\prime}$ and $\mathbf{O A}^{\prime}$ in Fig. 6 can be obtained from the closed pack of the seven spheres in Figs. 13a and b applying the cosine rule for triangle to Fig. 13b.

$$
\begin{aligned}
\cos \angle A^{\prime} O B^{\prime} & =\frac{\left|\mathbf{O} \mathbf{A}^{\prime}\right|^{2}+\left|\mathbf{O} \mathbf{B}^{\prime}\right|^{2}-\left|\mathbf{A}^{\prime} \mathbf{B}^{\prime}\right|^{2}}{2\left|\mathbf{O} \mathbf{A}^{\prime}\right|\left|\mathbf{O B} \mathbf{B}^{\prime}\right|} \\
& =\frac{2 d^{2}+2 d^{2}-(2 d)^{2}}{4 d^{2}}=0
\end{aligned}
$$

From Eq. 88, it is established that $\angle A^{\prime} O B^{\prime}=90^{\circ}$. This result from Fig. 13b, though a little complicated, is consistent with that obtained from Fig. 6 . The sphere $\mathrm{O}$ and the other six spheres in Fig. 13b form a unit of seven spheres. With these calculations, it has been shown that manipulating two such units can demonstrate how a face-centered cubic cell can be constructed from ABCA close-packing. With reference to Figs. 6 or $13 \mathrm{~b}$ it can be established how two units of seven OABCA' $B^{\prime} C^{\prime}$ spheres should be assembled into a face-centered cubic cell, i.e., $A, B$, and $C$ are at positions of face-centers and $O, A^{\prime}, B^{\prime}$, and $C^{\prime}$ are at the vertices of the cubic cell. The complicated calculation adopted here has its own merits since it provides an intuitive and a different perspective way to comprehend visually how a face-centered cubic cell can be extracted from $A B C$ packing, even though a simpler indirectly calculation has been shown by Fig. $13 \mathrm{c}$ where $|\mathbf{O C}|=\left|\mathbf{C B}^{\prime}\right|=\left|\mathbf{C A}^{\prime}\right|=d$ and $\left|\mathbf{O A}^{\prime}\right|=\left|\mathbf{O B}^{\prime}\right|$. 


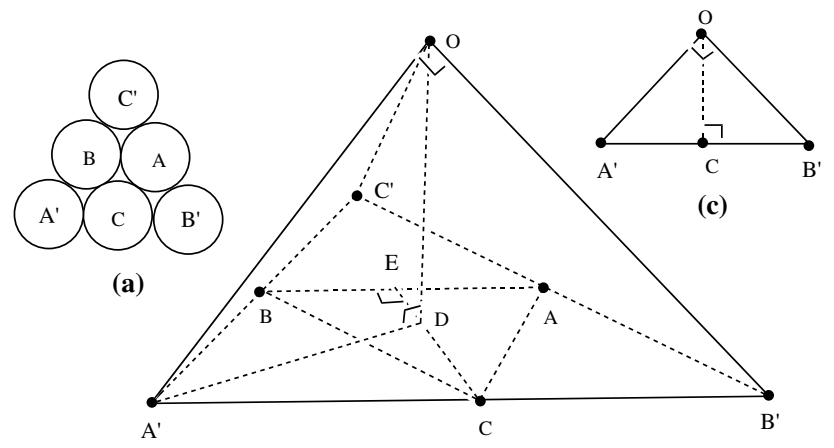

(b)

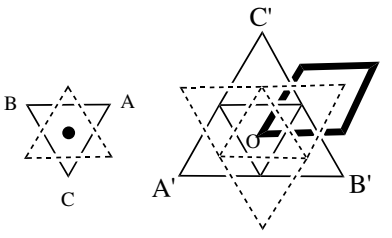

(d)

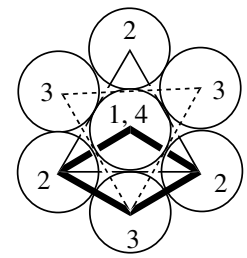

(f)
Fig. 13 The (111) plane in Fig. 6 is shown here as close-packed equal spheres by (a). The diameter of the sphere is $d$. If layer $A B C$ in (a) is horizontal, then sphere $O$ in (b) is positioned on spheres $A, B$, and $C$. OD is the distance from $O$ to the $A B C$ layer. Letters other than $D$ and $E$ represent the centers of spheres. $O A B C$ is a regular tetrahedron. The relationship between $A B C A$ packing and a face-centered cubic cell can be demonstrated by assembling two units of the seven spheres $O A B C A^{\prime} B^{\prime} C^{\prime}$ show from (b). It has been shown in (c) that the angle between $\mathbf{O A}^{\prime}$ and $\mathbf{O B}^{\prime}$ is $90^{\circ}$. (d, e) indicate $A B C$ packing and (f) indicates $A A A$ packing. d-f show that both the $A B C A$ packing and $A A A$ packing can be represented by either a rhombohedral lattice or a hexagonal lattice. $O$ in (d) is below $A B C$ layer. The numbers in (f) indicate layers at which the vertices of a rhombohedral cell is located. A hexagonal cell can be identified with the help of the bold lines shown in $(\mathbf{e}, \mathbf{f})$

The rhombohedral cell in Fig. 13d is primitive and a hexagonal cell with three nodes is indicated with bold line in Fig. 13e in ABCA packing. The relationships between the rhombohedral cell $h R$ and the hexagonal cell in ABCA packing can be drawn from (e) and (d). The vertices of a rhombohedral cell with three nodes are shown from a hexagonal AAAA packing $(h P)$ by Fig. $13 f$ with layer numbers to show where the vertices of this cell are located. A conventional primitive hexagonal cell with only one lattice node has been indicated from AAAA packing in Fig. $13 f$.

The simple and the face-centered cubic lattices can be related to each other from the $A B C$ packing by introducing some vacancies in the packing. Using this perspective, it is easy to see that the structure of spinel $\mathrm{MgAl}_{2} \mathrm{O}_{4}$ can be decomposed into several simple cubic units while the spinel $\mathrm{MgAl}_{2} \mathrm{O}_{4}$ can be described by a face-centered cubic structure packed with $\mathrm{O}^{2}, \mathrm{Al}^{3+}$ ions, and some vacancies [26]. The space group of spinel $\mathrm{MgAl}_{2} \mathrm{O}_{4}$ is the same as that of diamond.

\section{References}

1. Nespolo M (2015) The ash heap of crystallography: restoring forgotten basic knowledge. J Appl Cryst 48:1290-1298 See footnote 2

2. Nespolo M (2017) The ash heap of crystallography: restoring forgotten basic knowledge. Corrigendum. J Appl Cryst 50:978-979

3. Nespolo M (2017) Comments on the article 'Comparison of calculations for interplanar distances in a crystal lattice'. Crystallogr Rev 23(4):302-303

4. Fan Q (2016) A new method of calculating planar density: the position-duplication-number method. J Appl Cryst 49:1454-1458

5. Fan $\mathrm{Q}$ (2012) A new method of calculating interplanar spacing: the position-factor method. J Appl Cryst 45:1303-1308

6. Chambers C, Sumner P (2012) Replication is the only solution to scientific fraud. The Guardian. 14 September https://www. theguardian.com/commentisfree/2012/sep/14/solution-scien tific-fraud-replication

7. de Heer J (1957) The principle of Le Châtelier's and Braun. J Chem Educ 34:375-380

8. Gold J, Gold V (1984) Neither Le Châtelier's nor a principle. Chem Br 20:802-804

9. Liu Y, Liu Y, Drew MGB (2015) A mathematical approach to chemical equilibrium theory for gaseous systems-Part IV A mathematical Clarification of Le Châtelier's principle. J Math Chem 53(8):1835-1870

10. Katz L (1961) A systematic way to avoid Le Châtelier's principle in chemical reactions. J Chem Educ 38:375-377

11. Helfferich FG (1985) Le Châtelier-right or wrong? Optimizing chemical reaction equilibria. J Chem Educ 62:305-308

12. Liu Y, Liu Y, Drew MGB (2014) The handedness structure of octahedral metal complexes with chelating ligands. Coord Chem Rev 260:37-64

13. Liu Y, Liu B, Liu Y, Drew MGB (2011) Correlations between two sets of angular relation equations. J Math Chem 49:2089-2108

14. Liu Y, Liu Y, Liu B (2011) A new method for obtaining RussellSaunders terms. J Chem Educ 88(3):295-298

15. Liu Y, Liu Y, Drew MGB (2012) The use of three simple related procedures in determining the Russell-Saunders terms of equivalent electrons. Chem Educator 17:118-124

16. Schekman R (2013) How journals like Nature, Cell and Science are damaging science. The Guardian. 9 December https://www. theguardian.com/commentisfree/2013/dec/09/how-journalsnature-science-cell-damage-science

17. Liu Y, Drew MGB, Liu Y, Liu L (2013) Anodic polarization curves revisited. J Chem Educ 90(1):76-81

18. Liu Y, Drew MGB, Li H, Liu Y (2020) An experimental and theoretical investigation into methods concerned with "reflection loss" for microwave absorbing materials. Mater Chem Phys 243:122624

19. Liu Y, Lin Y, Zhao K, Drew MGB, Liu Y (2020) Microwave absorption properties of $\mathrm{Ag} / \mathrm{NiFe}_{2-x} \mathrm{Ce}_{x} \mathrm{O}_{4}$ characterized by an alternative procedure rather than the main stream method using reflection loss. Mater Chem Phys 243:122615

20. Liu Y, Zhao K, Drew MGB, Liu Y (2018) A theoretical and practical clarification on the calculation of reflection loss for microwave absorbing materials. AIP Adv 8:015223

21. Yang K, Liu Y, Drew MGB, Liu Y (2018) Preparation and characterization of $\mathrm{BaSm}_{x} \mathrm{Fe}_{12-x} \mathrm{O}_{19}$ /polypyrrole composites. J Mater Sci: Mater Electron 29(15):13148-13160

22. Liu Y, Drew MGB, Liu Y (2019) Characterization microwave absorption from active carbon / $\mathrm{BaSm}_{x} \mathrm{Fe}_{12-x} \mathrm{O}_{19}$ /polypyrrole composites analyzed with a more rigorous method. J Mater Sci: Mater Electron 30(2):1936-1956 
23. Hawkes SJ (2004) Chemistry is not a laboratory science. J Chem Educ 81(9):1257

24. Sacks LJ (2005) Reaction to 'Chemistry is not a laboratory science'. J Chem Educ 82(7):997

25. Nearly two years after a university asked for retractions, two journals have done nothing. http://retractionwatch .com/2018/07/13/nearly-two-years-after-a-university-asked -for-retractions-two-journals-have-done-nothing/

26. Liu Y, Jin J, Drew MGB, Liu Y (2017) Several theoretical perspectives of ferrite based materials-part 2: close packing model for crystal structure. J Supercond Nov Magn 30(10):2777-2789

27. Liu Y, Li Y, Liu Y, Yin H-S, Wang L-L, Sun K, Gao Y (2011) Structure information of barium hexaferrite and strategies for its syntheses. Appl Mech Mater 69:6-11

28. Liu Y, Drew MGB, Wang J, Zhang M, Liu Y (2010) Efficiency and purity control in the preparation of pure and/or aluminum doped barium ferrites by hydrothermal methods using ferrous ions as reactants. J Magn Magn Mater 322(3):366-374

29. Liu Y, Drew MGB, Liu Y (2011) Preparation and magnetic properties of barium ferrites substituted with manganese, cobalt and tin. J Magn Magn Mater 323:945-953

30. Liu Y, Liu Y, Drew MGB (2013) Correlation between Fourier series expansion and Hückel orbital theory. J Math Chem 51(2):503-531

31. Anonymous academic (2017) Plagiarism is rife in academia-so why is it rarely acknowledged. The Guardian, 27 October https ://www.theguardian.com/higher-education-network/2017/ oct/27/plagiarism-is-rife-in-academia-so-why-is-it-rarely-ackno wledged

32. Jha A (2012) False positives-fraud and misconduct are threatening scientific research. The Guardian, 13 September https:// www.theguardian.com/science/2012/sep/13/scientific-resea rch-fraud-bad-practice

33. Oransky I, Marcus A (2018) Harvard and the Brigham call for more than 30 retractions of cardiac stem cell research. STAT, October 14 https://www.statnews.com/2018/10/14/harvardbrigham-retractions-stem-cell/

34. Harvard calls for retraction of dozens of studies by noted cardiologist. New York Times, October 16, 2018. http://www.stara dvertiser.com/2018/10/16/news/harvard-calls-for-retraction -of-dozens-of-studies-by-noted-cardiologist/

35. Molkentin J (2018) There are no stem cells in the heart. Quit trying to publish those results. STAT, October 14 https://www.statn ews.com/2018/10/14/harvard-brigham-retractions-stem-cell/

36. Miller indices, Online Dictionary of Crystallography. https://dicti onary.iucr.org/Miller_indices

37. Kelly A, Knowles KM (2012) Crystallography and crystal defects, 2nd edn. Wiley, New Delhi, pp 469-472 Pondicherry

38. Chatterjee SK (2008) Crystallography and the world of symmetry. Springer, Berlin, pp 47-56

39. Liu Y, Liu Y, Drew MGB (2017) Comparison of calculations for interplanar distances in a crystal lattice. Cryst Rev 23:252-301

40. Schwarzenbach D (1996) Crystallography. Pinketon AA, translator. Wiley, New York, pp 2-7

41. Liu Y, Liu Y, Drew MGB (2020) Relationship between heat capacities derived by different but connected approaches. Am J Phys $88: 51-59$
42. Liu Y, Yu H, Drew MGB, Liu Y (2018) A systemized parameter set applicable to microwave absorption for ferrite based materials. J Mater Sci: Mater Electron 29(2):1562-1575

43. Borchardt-Ott W (2011) Crystallography, an introduction. R. O. Gould, translator, 3rd edn. Springer, London, pp 284-287

44. Woolfson MM (1997) An introduction to X-ray crystallography, 2nd edn. Cambridge University Press, New York, pp 67-74

45. Hahn T (2005) International tables for crystallography, Vol. A: space-group symmetry, 5th edn. Springer, Dordrecht, pp 659-697

46. Liu Y, Liu Y, Yin H, Drew MGB (2017) Several theoretical perspectives of ferrite-based materials-part 3: crystal structure and synthesis. J Supercond Nov Magn 30:3019-3025

47. Liu Y, Tai R, Drew MGB, Liu Y (2017) Several theoretical perspectives of ferrite-based materials-parts 1: transmission-line theory and microwave absorption. J Supercond Nov Magn 30:2489-2504

48. Vainshtein BK (1996) Fundamentals of crystals-symmetry, and methods of structural crystallography (modern crystallography) vol I, 2nd edn. Springer, Bangalore, p 15

49. Vainshtein BK, Fridkin VM, Indenbom VL (1995) Structure of crystals (modern crystallography). vol. 2, 2nd enlarged edn. Springer, Berlin, p 139, 349, 359, 362-363

50. https://en.wikipedia.org/wiki/Diamond_cubic

51. Hammond C (2009) The basics of crystallography and diffraction, 3rd edn. Oxford University Press, International Union of Crystallography, Oxford, pp 63-76

52. Desiraju GR (2003) Perspectives in supramolecular chemistry (crystal design—structure and function). vol 7. Wiley, Chichester, p. $82,84,90$

53. Dresselhaus MS, Dresselhaus G, Jorio A (2008) Group theory, application to the physics of condensed matter. Springer, Berlin, p 196, 210, 230-233, 250

54. Authier A (2003) Physical properties of crystals (international tables for crystallography), vol D. Kluwer Academic Publishers, London, p 446

55. Fischer W, Burzlaff H, Hellner E, Donnay JDH (1973) Space groups and lattice complexes. U.S. Department of Commerce, National Bureau of Standards, Washington, DC

56. Liu Y, Liu Y (2013) Several methods for solving the Zn-S bond length in cubic $\mathrm{ZnS}$ cell. Huaxue Jiaoxue (Educ Chem) (1), 70-72. http://www.cnki.com.cn/Article/CJFDTotal-HXJX201301031.htm (in Chinese)

57. Liu Y, Drew MGB, Liu Y, Liu Y, Cao FL (2014) A comparative study of $\mathrm{Fe}_{3} \mathrm{O}_{4}$ /polyaniline composites with octahedral and microspherical inorganic kernels. J Mater Sci 49:3694-3704

58. Tannous $C$ (2020) Crystal structure, $X$-ray diffraction and oblique geometry. Eur J Phys 41(1):015501

59. Deas HD, Hamill CM (1957) A note on the geometry of lattice planes. Acta Cryst 10:541-542

60. Jaswon MA, Dove DB (1955) The geometry of lattice planes I. Acta Cryst 8:88-91

Publisher's Note Springer Nature remains neutral with regard to jurisdictional claims in published maps and institutional affiliations. 